\title{
A GENERALIZATION OF THE LINE TRANSLATION THEOREM
}

\author{
JIAN WANG
}

\begin{abstract}
Through the method of brick decomposition and the operations on essential topological lines, we generalize the line translation theorem of Beguin, Crovisier and Le Roux (2006) in the case where the property of preserving a finite measure with total support is replaced by the intersection property.
\end{abstract}

\section{INTRODUCTION}

Let $\mathbb{A}=\mathbb{R} / \mathbb{Z} \times \mathbb{R}$ be the open annulus. We denote by $\pi$ the covering map

$$
\begin{aligned}
\pi: \mathbb{R}^{2} & \rightarrow \mathbb{A}, \\
(x, y) & \mapsto(x+\mathbb{Z}, y),
\end{aligned}
$$

and by $T$ the generator of the covering transformation group

$$
\begin{aligned}
T: \mathbb{R}^{2} & \rightarrow \mathbb{R}^{2}, \\
(x, y) & \mapsto(x+1, y) .
\end{aligned}
$$

Write respectively $S$ and $N$ for the lower and the upper end of $\mathbb{A}$. We call an essential line in $\mathbb{A}$ every simple path, parametrized by $\mathbb{R}$, properly embedded in $\mathbb{A}$, joining one end to the other one. We call an essential circle in $\mathbb{A}$ every simple closed curve which is not null-homotopic.

Let $f$ be a homeomorphism of $\mathbb{A}$. We say that $f$ satisfies the intersection property if any essential circle in $\mathbb{A}$ meets its image by $f$. We denote the space of all homeomorphisms of $\mathbb{A}$ which are isotopic to the identity as $\mathrm{Homeo}_{*}(\mathbb{A})$ and its subspace whose elements additionally have the intersection property as $\operatorname{Homeo}_{*}^{\wedge}(\mathbb{A})$. If $X$ is a topological space and $A$ is a subset of $X$, denote respectively by $\operatorname{Int}(A)$, $\mathrm{Cl}(A)$ and $\partial A$ the interior, the closure and the boundary of $A$.

The Arc Translation Theorem was first proved by Kwapisz $\mathrm{Kw}$ for diffeomorphisms of the closed annulus $\overline{\mathbb{A}}=\mathbb{R} / \mathbb{Z} \times[0,1]$ with some three-dimensional topology arguments in 2002. It asserts that for a diffeomorphism $f$ of $\overline{\mathbb{A}}$ which is isotopic to the identity and has a unique irrational rotation number $\alpha$ there exists, for every $n \in \mathbb{N}$, an arc that joins the two boundary components of $\overline{\mathbb{A}}$ and is disjoint from its first $n$ iterates. These iterates must then have the same combinatorics as $n$ iterates of a vertical segment $\{\theta\} \times[0,1]$ under the rigid rotation of angle $\alpha$. It was then reproduced by Béguin, Crovisier, Le Roux and Patou BCLP for homeomorphisms of the closed annulus by using two-dimensional topology techniques in 2004. In 2006,

Received by the editors September 14, 2011 and, in revised form, December 20, 2012 and December 26, 2012.

2010 Mathematics Subject Classification. Primary 37E45, 37E30.

The author was supported by CSC and SRF for ROCS, SEM. This is a part of the author's Ph.D. thesis at Tsinghua University. 
Béguin, Crovisier and Le Roux $\mathrm{BCL}$ proved an analogous version, the line translation theorem, for area preserving homeomorphisms on the open annulus. The goal of this paper is to generalize the line translation theorem in the case where the property of preserving a finite measure with total support is replaced by the intersection property. The reason why we consider the intersection property is that some interesting questions can be reduced to homeomorphisms of the open annulus which satisfy the intersection property but do not preserve a finite measure. For example, consider a homeomorphism $f$ of a closed surface $M$ with genus at least one that preserves a finite measure with total support. Take a lift $F$ to the universal covering space $\widetilde{M}$ (homeomorphic to the Poincaré disk) of $M$ and suppose that $F$ has a fixed point. When we remove this point, we obtain a map of the open annulus that satisfies the intersection property but does not preserve a finite measure. We will strongly use the arguments of Béguin, Crovisier, and Le Roux BCL] but will have to add some crucial lemmas to weaken the assumptions of their theorem. Let us first recall some results and then state our main results.

When $f \in \operatorname{Homeo}_{*}(\mathbb{A})$, we define the rotation number of a positively recurrent point as follows (see [Lec1 for details). We say that a positively recurrent point $z$ has a rotation number $\rho(F ; z) \in \mathbb{R}$ for a lift $F$ of $f$ to the universal covering space $\mathbb{R}^{2}$ of $\mathbb{A}$, if for every subsequence $\left\{f^{n_{k}}(z)\right\}_{k \geq 0}$ of $\left\{f^{n}(z)\right\}_{n \geq 0}$ which converges to $z$, we have

$$
\lim _{k \rightarrow+\infty} \frac{p_{1} \circ F^{n_{k}}(\widetilde{z})-p_{1}(\widetilde{z})}{n_{k}}=\rho(F ; z),
$$

where $\widetilde{z} \in \pi^{-1}(z)$ and $p_{1}$ is the first projection $p_{1}(x, y)=x$. In particular, the rotation number $\rho(F ; z)$ always exists and is rational when $z$ is a fixed or periodic point of $f$. Let $\operatorname{Rec}^{+}(f)$ be the set of positively recurrent points of $f$. We denote the set of rotation numbers of positively recurrent points of $f$ as $\operatorname{Rot}(F)$.

It is well known that a positively recurrent point of $f$ is also a positively recurrent point of $f^{q}$ for all $q \in \mathbb{N}$ (we give a proof in the Appendix; see Lemma 19). By the definition of a rotation number, we easily get that $\operatorname{Rot}(F)$ satisfies the following elementary properties:

1. $\operatorname{Rot}\left(T^{k} \circ F\right)=\operatorname{Rot}(F)+k$ for every $k \in \mathbb{Z}$;

2. $\operatorname{Rot}\left(F^{q}\right)=q \operatorname{Rot}(F)$ for every $q \in \mathbb{N}$.

We recall that a Farey interval is an interval of the form $] \frac{p}{q}, \frac{p^{\prime}}{q^{\prime}}\left[\right.$ with $q, q^{\prime} \in \mathbb{N} \backslash\{0\}$, $p, p^{\prime} \in \mathbb{Z}$ and $q p^{\prime}-p q^{\prime}=1$. Our main result is the following:

Theorem 1 (Generalization of the line translation theorem). Let $f \in \operatorname{Homeo}_{*}^{\wedge}(\mathbb{A})$ and $F$ be a lift of $f$ to $\mathbb{R}^{2}$. Assume that $\operatorname{Rot}(F) \neq \emptyset$ and its closure is contained in a Farey interval $] \frac{p}{q}, \frac{p^{\prime}}{q^{\prime}}[$. Then, there exists an essential line $\gamma$ in $\mathbb{A}$ such that the lines $\gamma, f(\gamma), \cdots, f^{q+q^{\prime}-1}(\gamma)$ are pairwise disjoint. Moreover, the cyclic order of these lines is the same as the cyclic order of the $q+q^{\prime}-1$ first iterates of a vertical line $\{\theta\} \times \mathbb{R}$ under the rigid rotation with angle $\rho$, for any $\rho \in] \frac{p}{q}, \frac{p^{\prime}}{q^{\prime}}[$.

It is easy to see that a homeomorphism of $\mathbb{A}$ that preserves a finite measure with total support satisfies the intersection property. Note that the statement of Theorem 1 with this stronger condition is exactly what is done in BCL. The first similar result stated above is an analogue of a theorem of Kwapisz dealing with diffeomorphisms of the two-torus $\mathrm{Kw}$. In his proof, Kwapisz introduces the suspension of the diffeomorphism under consideration and uses some threedimensional topology techniques to find the required curve as the intersection of 
two cross sections of this suspension. The proofs of $[\mathrm{BCL}]$ and this paper are purely two-dimensional.

Let us consider the simple case of Theorem 1 when $(p, q)=(0,1)$ and $\left(p^{\prime}, q^{\prime}\right)=$ $(1,1)$. We obtain the following corollary.

Corollary 2. Let $f \in \operatorname{Homeo}_{*}^{\wedge}(\mathbb{A})$. We suppose that $F$ is a lift of $f$ to $\mathbb{R}^{2}$ and that

$$
\emptyset \neq \mathrm{Cl}(\operatorname{Rot}(F)) \subset] 0,1[\text {. }
$$

Then there exists an essential line in $\mathbb{A}$ that is free for $f$, that means disjoint from its image by $f$.

A similar result in the case when $\mathbb{A}$ is a closed annulus is known due to Bonatti and Guillou G2 : if $f$ is a homeomorphism of the closed annulus $\mathbb{R} / \mathbb{Z} \times[0,1]$ isotopic to the identity and fixed point free, then either there is a free simple path for $f$ that joins the two boundary of the closed annulus or there is a free essential circle in the closed annulus for $f$.

As we will recall later, the rotation set of $F$ is a closed interval if $f$ satisfies the intersection property and $F$ is any lift of $f$ to the universal covering space $\mathbb{R} \times[0,1]$. Therefore, if the map $f$ has no fixed point, we can find a lift $F$ of $f$ to $\mathbb{R} / \mathbb{Z} \times[0,1]$ such that the rotation number set of $F$ is contained in ]0,1[.

As an immediate corollary of Theorem 1 we get the following generalization of the Corollary 0.3 in BCL]:

Corollary 3. We suppose that $f \in \operatorname{Homeo}_{*}^{\wedge}(\mathbb{A})$ has a rotation set reduced to a single irrational number $\rho$ (for any given lift $F$ of $f$ ). Then, for every $n \in \mathbb{N} \backslash\{0\}$, there exists an essential line $\gamma$ in $\mathbb{A}$, such that the lines $\gamma, f(\gamma), \cdots, f^{n}(\gamma)$ are pairwise disjoint. The cyclic order of the lines $\gamma, f(\gamma), \cdots, f^{n}(\gamma)$ is the same as the cyclic order of the $n$ first iterates of a vertical line under the rigid rotation of angle $\rho$.

Similarly to the argument of Theorem 0.4 in BCL, we have the following corollary:

Corollary 4. We suppose that $f \in \operatorname{Homeo}_{*}^{\wedge}(\mathbb{A})$ has a rotation set reduced to a single irrational number $\rho$ (for any given lift $F$ of $f$ ). The rigid rotation of angle $\rho$ is in the closure (for the compact-open topology) of the conjugacy class of $f$.

Note here that one does not know if $f$ is in the closure of the conjugacy class of a rigid rotation of angle $\rho$.

Theorem 1 above is a global result on the open annulus. A local version was studied by Patou in her thesis $[\mathrm{P}$ ] under similar hypotheses of the line translation theorem in BCL. More precisely, she considered a local homeomorphism $F$ between two neighborhoods of the origin $O$ in $\mathbb{R}^{2}$ that fixes $O$. If $F$ preserves the orientation and the area of $\mathbb{R}^{2}$, and it has no other periodic point except $O$ in its domain, then for every $N \in \mathbb{N}$, there exists a simple arc $\Gamma$ in a small neighborhood of $O$ that issues from $O$ such that the $\operatorname{arcs} \Gamma, F(\Gamma), \cdots, F^{N}(\Gamma)$ are pairwise disjoint except at $O$.

We will introduce some mathematical objects and recall some well-known facts in Section 2. In Section 3, we first state some crucial lemmas without proof and then prove Theorem 1. In Section 4, we prove the lemmas stated in Section 3, and we give some remarks about the relations between the positively recurrent set and the rotation number set. In Section 5, we define a weak rotation number which 
is a generalization of the rotation number we have defined above. We prove the generalization of the line translation theorem in the weak sense. Finally, in Section 6 , we provide a proof of some well-known facts that we require in this paper but were unable to find in the literature.

\section{Preliminaries}

2.1. Essential topological lines in $\mathbb{R}^{2}$. A topological line in $\mathbb{R}^{2}$ is the image of a proper continuous embedding of $\mathbb{R}$. Equivalently, using the Schoenflies theorem, it is the image of a Euclidean line under a homeomorphism of $\mathbb{R}^{2}$. Let $\Gamma$ be a topological line whose orientation is induced by a parametrization. We denote by $L(\Gamma)$ the connected component of $\mathbb{R}^{2} \backslash \Gamma$ on the left of $\Gamma$, and by $R(\Gamma)$ the connected component of $\mathbb{R}^{2} \backslash \Gamma$ on the right of $\Gamma$. We get a partial order relation $\leq$ on the set of oriented lines by writing: $\Gamma_{1} \leq \Gamma_{2}$ if $L\left(\Gamma_{1}\right) \subset L\left(\Gamma_{2}\right)$ (or equivalently $R\left(\Gamma_{2}\right) \subset$ $\left.R\left(\Gamma_{1}\right)\right)$. We also get a transitive relation $<$ by writing $\Gamma_{1}<\Gamma_{2}$ if $\mathrm{Cl}\left(L\left(\Gamma_{1}\right)\right) \subset L\left(\Gamma_{2}\right)$ (or equivalently $\left.\mathrm{Cl}\left(R\left(\Gamma_{2}\right)\right) \subset R\left(\Gamma_{1}\right)\right)$.

We call an essential line in $\mathbb{R}^{2}$ an oriented line $\Gamma$ such that $\lim _{t \rightarrow+\infty} p_{2}(\Gamma(t))=$ $+\infty$ and $\lim _{t \rightarrow-\infty} p_{2}(\Gamma(t))=-\infty$, where $p_{2}(x, y)=y$ and $t \mapsto \Gamma(t)$ is a parametrization. If $\Gamma$ is a line and $F$ is a homeomorphism of $\mathbb{R}^{2}$, then $F(\Gamma)$ is a line. Moreover, if $\Gamma$ is oriented, then there is a natural orientation for $F(\Gamma)$. We say that an orientation preserving homeomorphism $F$ of $\mathbb{R}^{2}$ is essential if for every essential line $\Gamma$ in $\mathbb{R}^{2}, F(\Gamma)$ is also an essential line in $\mathbb{R}^{2}$. Any homeomorphism $F$ that lifts a homeomorphism $f$ of an open annulus $\mathbb{A}$ isotopic to the identity is an essential homeomorphism.

Let $\Gamma_{1}$ and $\Gamma_{2}$ be two essential lines in $\mathbb{R}^{2}$, and let $U$ be the unique connected component of the set $L\left(\Gamma_{1}\right) \cap L\left(\Gamma_{2}\right)$ which contains half lines of the form $]-\infty, a[\times\{b\}$ for some numbers $a$ and $b$. Then the boundary of $U$ is an essential line in $\mathbb{R}^{2}$, denoted by $\Gamma_{1} \vee \Gamma_{2}$ (the proof of this fact uses a classical result by B. Kerékjártó [Ke. See [BCLP] and $\mathrm{BCL}]$.

Remark 1. Let $\Gamma_{1}, \Gamma_{2}, \Gamma_{3}$ be three essential lines in $\mathbb{R}^{2}$. The following properties are immediate consequences of the definition of the line $\Gamma_{1} \vee \Gamma_{2}$.

(1) The line $\Gamma_{1} \vee \Gamma_{2}$ is included in the union of the lines $\Gamma_{1}$ and $\Gamma_{2}$. Hence, if $\Gamma_{3}<\Gamma_{1}$ and $\Gamma_{3}<\Gamma_{2}$, then $\Gamma_{3}<\Gamma_{1} \vee \Gamma_{2}$.

(2) The sets $R\left(\Gamma_{1}\right)$ and $R\left(\Gamma_{2}\right)$ are included in the set $R\left(\Gamma_{1} \vee \Gamma_{2}\right)$. In other words, we have $\Gamma_{1} \vee \Gamma_{2} \leq \Gamma_{1}$ and $\Gamma_{1} \vee \Gamma_{2} \leq \Gamma_{2}$.

2.2. Brouwer theory. A Brouwer homeomorphism is an orientation preserving fixed point free homeomorphism of $\mathbb{R}^{2}$. Given a Brouwer homeomorphism $F$, a Brouwer line for $F$ is a topological line $\Gamma$, disjoint from $F(\Gamma)$ and separating $F(\Gamma)$ from $F^{-1}(\Gamma)$. The Brouwer Plane Translation Theorem asserts that every point belongs to a Brouwer line.

Now we introduce the following "equivariant" version of the Brouwer Plane Translation Theorem which has been proved by Guillou and Sauzet:

Theorem $5([\mathrm{G} 1],[\mathrm{S}])$. Let $f \in \operatorname{Homeo}_{*}(\mathbb{A})$ and $F$ be a lift of $f$ to $\mathbb{R}^{2}$. If $F$ is fixed point free, then there exists an essential circle in $\mathbb{A}$ that is free under $f$ (and therefore lifts to a Brouwer line of $F$ ) or there exists an essential line in $\mathbb{A}$ that lifts to a Brouwer line of $F$.

Let $\Gamma$ be a Brouwer line. It can be oriented such that $\Gamma<F(\Gamma)$. Since $F$ preserves the orientation, we have $F^{k}(\Gamma)<F^{k+1}(\Gamma)$. By induction, we see that 
$F^{p}(\Gamma)<F^{q}(\Gamma)$ if and only if $p<q$. In particular, the lines $\left(F^{k}(\Gamma)\right)_{k \in \mathbb{Z}}$ are pairwise disjoint.

Now let $U$ be the open region of $\mathbb{R}^{2}$ situated between the lines $\Gamma$ and $F(\Gamma)$, and $\mathrm{Cl}(U)=\Gamma \cup U \cup F(\Gamma)$. The sets $\left(F^{k}(U)\right)_{k \in \mathbb{Z}}$ are pairwise disjoint. As a consequence, the restriction of $F$ to the open set $O_{U}=\bigcup_{k \in \mathbb{Z}} F^{k}(\mathrm{Cl}(U))$ is conjugate to a translation. In particular, if the iterates of $\mathrm{Cl}(U)$ cover the whole plane, then $F$ itself is conjugate to a translation.

2.3. Franks' Lemma and brick decompositions. A free disk chain for a homeomorphism $F$ of $\mathbb{R}^{2}$ is a finite set $b_{i}(i=1,2, \cdots, n)$ of embedded open disks in $\mathbb{R}^{2}$ satisfying

(1) $F\left(b_{i}\right) \cap b_{i}=\emptyset$ for $1 \leq i \leq n$;

(2) if $i \neq j$, then either $b_{i}=b_{j}$ or $b_{i} \cap b_{j}=\emptyset$;

(3) for $1 \leq i \leq n$, there exists $m_{i}>0$ such that $F^{m_{i}}\left(b_{i}\right) \cap b_{i+1} \neq \emptyset$.

We say that $\left(b_{i}\right)_{i=1}^{n}$ is a periodic free disk chain if $b_{1}=b_{n}$.

In F1, Franks proved the following useful lemma about the existence of fixed points of an orientation preserving homeomorphism $F$ of $\mathbb{R}^{2}$ from Brouwer theory.

Proposition 6 (Franks' Lemma). Let $F: \mathbb{R}^{2} \rightarrow \mathbb{R}^{2}$ be an orientation preserving homeomorphism which possesses a periodic free disk chain. Then $F$ has at least one fixed point.

A brick decomposition of a surface $S$ (not necessarily closed) is given by a one-dimensional stratified set $\Sigma$ (the skeleton of the decomposition) with a zerodimensional submanifold $V$ such that any vertex $v \in V$ is locally the extremity of exactly three edges. A brick is the closure of a connected component of $S \backslash \Sigma$. Write $\mathfrak{B}$ for the set of bricks. For any $\mathfrak{X} \subset \mathfrak{B}$ the union of bricks which are in $\mathfrak{X}$ is a sub-surface of $S$ with boundary. Suppose that $F$ is a homeomorphism of $S$. We can define the relation $B \mathscr{R} B^{\prime} \Leftrightarrow F(B) \cap B^{\prime} \neq \emptyset$ where $B, B^{\prime} \in \mathfrak{B}$, and write $B \preceq B^{\prime}$ if there exists a sequence $\left(B_{i}\right)_{0<i<n} \subset \mathfrak{B}$ such that $B_{0}=B, B_{n}=B^{\prime}$ and $B_{i} \mathscr{R} B_{i+1}$ for every $i \in\{0, \ldots, n-1\}$. The union $B_{\succeq}=\bigcup_{B^{\prime} \succeq B} B^{\prime}$ (resp. $B_{\preceq}=\bigcup_{B^{\prime} \preceq B} B^{\prime}$ ) is a closed subset satisfying $F\left(B_{\succ}\right) \subset \operatorname{Int}\left(B_{\succ}\right)\left(\operatorname{resp} . F^{-1}\left(B_{\preceq}\right) \subset \operatorname{Int}\left(B_{\preceq}\right)\right)$.

Suppose that $S=\mathbb{R}^{2}$ and $F$ is an orientation preserving homeomorphism of $\mathbb{R}^{2}$ without fixed point; we say that $\mathfrak{B}$ is free if every brick $B \in \mathfrak{B}$ is free. The stronger version of Franks' Lemma given in Guillou and Le Roux [Ler1] asserts that there is no closed chain of bricks of $\mathfrak{B}$ if $\mathfrak{B}$ is free. This implies that $\preceq$ is a partial order. Furthermore, we can construct a maximal free decomposition: it is a brick decomposition with free bricks such that the union of two adjacent bricks is no more free [S]. The decomposition being maximal, two adjacent bricks are comparable. In fact, it appears that for every brick $B$, the union of bricks $B^{\prime} \succeq B$ adjacent to $B$ is non-empty, as is the union of adjacent bricks $B^{\prime} \preceq B$. This implies that $B_{\succ}$ and $B \preceq$ are connected surfaces with boundary. The fact that we are working with bricks implies that $\partial\left(B_{\succ}\right)$ and $\partial\left(B_{\preceq}\right)$ is a one-dimensional manifold; the inclusion $F\left(B_{\succeq}\right) \subset \operatorname{Int}\left(B_{\succeq}\right)$ implies that every component of $\partial\left(B_{\succeq}\right)$ and $\partial\left(B_{\preceq}\right)$ is a Brouwer line. If $F$ commutes with $T$, we can further assume that the free decomposition $\mathfrak{B}$ is invariant by $T$ (see Lec2 for details). The relation $\preceq$ is $T$-equivalent: $B \preceq B^{\prime} \Leftrightarrow T(B) \preceq T\left(B^{\prime}\right)$. However, a $T$-invariant maximal free decomposition does not necessarily have to exist. For example, there is no $T$-invariant free decomposition for $T^{k}(k \geq 3)$ which is maximal among the free decompositions (see $\underline{S}$ for details). Thus in this case, we cannot assert that two 
adjacent bricks are comparable and that $B_{\succeq}$ and $B_{\preceq}$ are connected. But if there exists $p \in \mathbb{Z} \backslash\{0\}$ such that $T^{p}(B) \in B_{\succeq}\left(\operatorname{resp} .{ }^{p}(B) \in T_{\preceq}\right)$, then the set $\bigcup_{k \in \mathbb{Z}} T^{k}\left(B_{\succeq}\right)$ (resp. $\bigcup_{k \in \mathbb{Z}} T^{k}\left(B_{\preceq}\right)$ ) is a closed connected surface with boundary, which is a direct consequence of Proposition 2.7 in Lec2. Moreover, the image by $F\left(\right.$ resp. $\left.F^{-1}\right)$ of $\bigcup_{k \in \mathbb{Z}} T^{k}\left(B_{\succeq}\right)$ (resp. $\bigcup_{k \in \mathbb{Z}} T^{k}\left(B_{\preceq}\right)$ ) is contained in its interior.

2.4. Le Calvez-Yoccoz's theorem. Recall that a Jordan curve in $\mathbb{R}^{2}$ is a compact subset that is homeomorphic to $S^{1}$ and a Jordan domain is the bounded connected components of $\mathbb{R}^{2} \backslash C$ where $C$ is a Jordan curve.

Suppose that $W$ and $W^{\prime}$ are two neighborhoods of 0 in $\mathbb{R}^{2}$ and $F: W \rightarrow W^{\prime}$ is a homeomorphism that fixes 0 . A neighborhood $U$ of 0 whose closure is in $W$ is an isolating set for $F$ if

$$
\bigcap_{n \in \mathbb{Z}} F^{-n}(\mathrm{Cl}(U)) \subset U .
$$

When $U$ is a Jordan domain, we say it is an isolating Jordan domain.

If $F$ has a unique fixed point 0 and has no isolating Jordan domain that contains 0 , by the definition, we have that any Jordan domain $U$ that contains 0 and whose closure is in $W$ satisfies

$$
\bigcap_{n \in \mathbb{Z}} F^{-n}(\mathrm{Cl}(U)) \cap \partial U \neq \emptyset
$$

Obviously, the connected component of $\bigcap_{n \in \mathbb{Z}} F^{-n}(\mathrm{Cl}(U))$ that contains 0 is a nontrivial invariant continuum $K$ of $F$.

Suppose now that $F$ has a unique fixed point 0 . Choose a number $r>0$ such that the ball $B_{r}=\left\{(x, y) \in \mathbb{R}^{2}||(x, y) \mid \leq r\right\}$ is in $W$. Define the Lefschetz index $i(F, 0)$ of $F$ at 0 as the degree of the map

$$
s \mapsto \frac{F(\gamma(s))-\gamma(s)}{|F(\gamma(s))-\gamma(s)|},
$$

where $\gamma$ is a loop in $W \backslash\{0\}$ homotopic to $\partial B_{r}$ and $s \mapsto \gamma(s)$ is a parametrization defined on the unit circle $\mathbf{S}^{1}$.

We will use the following important theorem LecY] later:

Theorem 7 (Le Calvez-Yoccoz's theorem). Suppose that $W$ and $W^{\prime}$ are two neighborhoods of 0 in $\mathbb{R}^{2}$ and $F: W \rightarrow W^{\prime}$ is a homeomorphism that fixes 0 , preserves the orientation and satisfies the following properties:

(1) F has no other periodic point except for 0 ;

(2) there is no neighborhood $V \subset W$ of 0 such that either $F(V) \subset V$ or $V \subset$ $F(V)$;

(3) there exists a neighborhood $V$ of 0 that is an isolating Jordan domain for $F$.

Then there exist positive integers $r$ and $q$ such that, for each $k \neq 0$, the Lefschetz index $i\left(F^{k}, 0\right)$ is equal to 1 if $k \notin q \mathbb{Z}$ and equal to $1-r q \leq 0$ if $k \in q \mathbb{Z}$.

\section{Proof of the generalization of the line translation theorem}

In this section, we first state some crucial lemmas and then prove the main result of Theorem 1. We delay the proofs of these lemmas to the next section. 
Lemma 8. Let $H_{1}, H_{2}, \ldots, H_{p}$ be pairwise commuting essential homeomorphisms of $\mathbb{R}^{2}$ and $\left(q_{i}\right)_{1 \leq i \leq p}$ be a family of positive integers. If there is an essential line $\Gamma$ in $\mathbb{R}^{2}$ such that $\Gamma<H_{i}^{q_{i}}(\Gamma)$ for every $i \in\{1, \cdots, p\}$, then there is an essential line $\Gamma^{\prime}$ in $\mathbb{R}^{2}$ such that $\Gamma^{\prime}<H_{i}\left(\Gamma^{\prime}\right)$ for every $i \in\{1, \cdots, p\}$.

Lemma 9. If $f \in \operatorname{Homeo}_{*}^{\wedge}(\mathbb{A})$, then $f^{q} \in \operatorname{Homeo}_{*}^{\wedge}(\mathbb{A})$ for any integer $q>1$.

Lemmas 8 and 9 are similar to Proposition 3.1 and Lemma 6.2 in [BCLP], respectively. In contrast to this, the following lemma is a key and new result given in this paper, by which we can directly prove Corollary 2 and further prove Theorem 1

Lemma 10. Let $f \in \operatorname{Homeo}_{*}^{\wedge}(\mathbb{A})$. We suppose that $F$ is a lift of $f$ to $\mathbb{R}^{2}$ and that

$$
\emptyset \neq \operatorname{Rot}(F) \subset[-q+2, q-2],
$$

where $q \geq 2$. Then there exists an oriented essential line $\gamma$ in $\mathbb{A}$ joining $S$ to $N$ that is lifted to an essential line $\Gamma$ in $\mathbb{R}^{2}$ which satisfies

$$
T^{-q}(\Gamma)<F(\Gamma)<T^{q}(\Gamma) .
$$

Proof of Theorem 1. First, we prove the special case where $(p, q)=(0,1)$ and $\left(p^{\prime}, q^{\prime}\right)=(1,1)$, that is, Corollary 2 . By the hypothesis, we can suppose that

$$
\operatorname{Rot}(F) \subset\left[\frac{1}{n}, 1-\frac{1}{n}\right]
$$

for some $n \in \mathbb{N}$ large enough. Hence the closure of $\operatorname{Rot}\left(F^{2 n} \circ T^{-n}\right)$ is contained in $[-n+2, n-2]$. The map $f$ satisfies the intersection property, thus does $f^{2 n}$ by Lemma 9. By Lemma 10, there exists an oriented essential line $\gamma$ in $\mathbb{A}$, joining $S$ to $N$, that is lifted to an essential line $\Gamma$ in $\mathbb{R}^{2}$ which satisfies

$$
T^{-n}(\Gamma)<\left(F^{2 n} \circ T^{-n}\right)(\Gamma)<T^{n}(\Gamma) .
$$

This implies that $\Gamma<F^{2 n}(\Gamma)<T^{2 n}(\Gamma)$.

By Lemma 8, let $H_{1}=F, H_{2}=F^{-1} \circ T$ and $q_{1}=q_{2}=2 n$. We can construct an essential line $\Gamma^{\prime}$ in $\mathbb{R}^{2}$ which satisfies $\Gamma^{\prime}<F\left(\Gamma^{\prime}\right)$ and $\Gamma^{\prime}<\left(F^{-1} \circ T\right)\left(\Gamma^{\prime}\right)$, so $\Gamma^{\prime}<F\left(\Gamma^{\prime}\right)<T\left(\Gamma^{\prime}\right)$. We deduce that $\gamma^{\prime}=\pi\left(\Gamma^{\prime}\right)$ is an essential line in $\mathbb{A}$ satisfying the conclusion of Corollary 2 .

Secondly, we turn to prove the general case. Let $\Phi=T^{-p} \circ F^{q}$ and $\Psi=T^{p^{\prime}} \circ F^{-q^{\prime}}$. In Proposition 4.1 of [BCLP, Béguin, Crovisier, Le Roux and Patou proved the following result:

"Let $\gamma$ be an essential line in the annulus $\mathbb{A}$. Assume that some lift $\Gamma$ of $\gamma$ is disjoint from its images under the maps $\Phi$ and $\Psi$. Then the $q+q^{\prime}-1$ first iterates of $\gamma$ under $f$ are pairwise disjoint, and ordered as the $q+q^{\prime}-1$ first iterates of a vertical line under a rigid rotation of angle $\rho \in] \frac{p}{q}, \frac{p^{\prime}}{q^{\prime}}[. "$

Remark here that the proof of $\mathrm{BCLP}$ is written in the context of the closed annulus, but it also works in the open annulus setting.

Therefore, to prove the theorem, it is enough to find an essential line $\gamma$ in $\mathbb{A}$, and a lift of $\gamma$ which is disjoint from its images under $\Phi$ and $\Psi$.

By the properties of rotation number, we have that $\left.\operatorname{Cl}\left(\operatorname{Rot}\left(F^{q q^{\prime}} \circ T^{-p q^{\prime}}\right)\right) \subset\right] 0,1[$. By Lemma 9. $f^{q q^{\prime}}$ satisfies the intersection property since $f$ satisfies this property. By Corollary 2 that we have proved above, there exists an oriented essential line $\gamma^{\prime}$ in $\mathbb{A}$ that is lifted to an essential line $\Gamma^{\prime}$ in $\mathbb{R}^{2}$ which satisfies

$$
\Gamma^{\prime}<\left(F^{q q^{\prime}} \circ T^{-p q^{\prime}}\right)\left(\Gamma^{\prime}\right)<T\left(\Gamma^{\prime}\right) .
$$


In particular, we have that $\Gamma^{\prime}<\left(F^{q} \circ T^{-p}\right)^{q^{\prime}}\left(\Gamma^{\prime}\right)=\Phi^{q^{\prime}}\left(\Gamma^{\prime}\right)$ and $\Gamma^{\prime}<T\left(\Gamma^{\prime}\right)$. Applying $T^{p q^{\prime}-q p^{\prime}}$ to formula (3.0.1) and observing that $q p^{\prime}-p q^{\prime}=1$, we get

$$
T^{-1}\left(\Gamma^{\prime}\right)<\left(F^{q q^{\prime}} \circ T^{-q p^{\prime}}\right)\left(\Gamma^{\prime}\right)<\Gamma^{\prime} .
$$

In particular, we have that $\Gamma^{\prime}<\left(F^{-q^{\prime}} \circ T^{p^{\prime}}\right)^{q}\left(\Gamma^{\prime}\right)=\Psi^{q}\left(\Gamma^{\prime}\right)$.

By Lemma 8, let $H_{1}=\Phi, H_{2}=\Psi, H_{3}=T, q_{1}=q^{\prime}, q_{2}=q$ and $q_{3}=1$. We can construct an essential line $\Gamma^{\prime \prime}$ in $\mathbb{R}^{2}$ which satisfies $\Gamma^{\prime \prime}<\Phi\left(\Gamma^{\prime \prime}\right), \Gamma^{\prime \prime}<\Psi\left(\Gamma^{\prime \prime}\right)$ and $\Gamma^{\prime \prime}<T\left(\Gamma^{\prime \prime}\right)$. Therefore, the essential line $\gamma^{\prime \prime}=\pi\left(\Gamma^{\prime \prime}\right)$ satisfies the conclusion of the theorem. We have completed the proof.

\section{Proofs of the LEMmas}

Proof of Lemma 8. Let us say that the essential line $\Gamma$ is of type $\left(q_{1}, q_{2}, \ldots, q_{p}\right)$ if $\Gamma<H_{i}^{q_{i}}(\Gamma)$ for every $i \in\{1, \ldots, p\}$. We want to prove that the existence of an essential line $\Gamma$ of type $\left(q_{1}, q_{2}, \ldots, q_{p}\right)$ implies the existence of an essential line $\Gamma^{\prime}$ of type $(1, \ldots, 1)$. By a simple induction argument, it is sufficient to prove the existence of an essential line $\Gamma^{\prime}$ of type $\left(1, q_{2}, \ldots, q_{p}\right)$.

We choose some essential lines of $\left(\Gamma_{i}\right)_{0 \leq i \leq q_{1}-1}$ in $\mathbb{R}^{2}$ such that

$$
\Gamma=\Gamma_{0}<\Gamma_{1}<\cdots<\Gamma_{q_{1}-1}<H_{j}^{q_{j}}\left(\Gamma_{0}\right), \quad \text { for every } j \in\{1, \ldots, p\} .
$$

Consider the essential line

$$
\Gamma^{\prime}=H_{1}^{q_{1}}\left(\Gamma_{0}\right) \vee H_{1}^{q_{1}-1}\left(\Gamma_{1}\right) \vee \cdots \vee H_{1}\left(\Gamma_{q_{1}-1}\right)=\bigvee_{i=0}^{q_{1}-1} H_{1}^{q_{1}-i}\left(\Gamma_{i}\right) .
$$

For every $i \in\left\{0, \ldots, q_{1}-2\right\}$, we have $\Gamma^{\prime} \leq H_{1}^{q_{1}-i}\left(\Gamma_{i}\right)$ (by the definition of $\Gamma^{\prime}$ and by item (2) of Remark 11) and $H_{1}^{q_{1}-i}\left(\Gamma_{i}\right)<H_{1}^{q_{1}-i}\left(\Gamma_{i+1}\right)$. Hence for every $i \in\left\{0, \ldots, q_{1}-2\right\}$, we get $\Gamma^{\prime}<H_{1}^{q_{1}-i}\left(\Gamma_{i+1}\right)$. Moreover, we have $\Gamma^{\prime} \leq H_{1}\left(\Gamma_{q_{1}-1}\right)$ and $\Gamma_{q_{1}-1}<H_{1}^{q_{1}}\left(\Gamma_{0}\right)$. Hence, $\Gamma^{\prime}<H_{1}^{q_{1}+1}\left(\Gamma_{0}\right)$. Finally, using item (1) of Remark 1] we get

$$
\Gamma^{\prime}<\bigvee_{i=0}^{q_{1}-1} H_{1}^{q_{1}-i+1}\left(\Gamma_{i}\right)=H_{1}\left(\Gamma^{\prime}\right) .
$$

Observe that $\Gamma_{i}<H_{j}^{q_{j}}\left(\Gamma_{i}\right)$ for every $i \in\left\{0,1, \cdots, q_{1}-1\right\}$ and $j \in\{2, \ldots, p\}$. Therefore, we have $\Gamma^{\prime} \leq H_{1}^{q_{1}-i}\left(\Gamma_{i}\right)<H_{1}^{q_{1}-i}\left(H_{j}^{q_{j}}\left(\Gamma_{i}\right)\right)$. Using the definition of $\Gamma^{\prime}$, the fact that $H_{j}$ commutes with $H_{1}$, and Remark 1, we have

$$
\Gamma^{\prime}<\bigvee_{i=0}^{q-1} H_{1}^{q_{1}-i}\left(H_{j}^{q_{j}}\left(\Gamma_{i}\right)\right)=H_{j}^{q_{j}}\left(\Gamma^{\prime}\right)
$$

Hence, the essential line $\Gamma^{\prime}$ is of type $\left(1, q_{2}, \ldots, q_{p}\right)$. We have completed the proof.

Proof of Lemma 9. It is similar to the proof of Lemma 8, For every essential circle $\gamma$ in $\mathbb{A}$, we denote by $B(\gamma)$ the connected component of $\mathbb{A} \backslash \gamma$ which is "below $\gamma$ " (that is, "containing" the lower end $S$ ). Given two essential circles $\gamma$ and $\gamma^{\prime}$ in $\mathbb{A}$, the boundary of the connected component of $B(\gamma) \cap B\left(\gamma^{\prime}\right)$ "containing" $S$ is an essential circle, which we denote by $\gamma \vee \gamma^{\prime}$. We write $\gamma<\gamma^{\prime}$ if $\mathrm{Cl}(B(\gamma)) \subset B\left(\gamma^{\prime}\right)$. 
To prove by contradiction, we suppose that there exists a positive integer $q$ and an essential circle $\gamma$ such that $\gamma<f^{q}(\gamma)$ (by replacing $f$ by $f^{-1}$ if necessary). We consider some essential circles $\left(\gamma_{i}\right)_{0 \leq i \leq q-1}$ such that

$$
\gamma=\gamma_{0}<\gamma_{1}<\cdots<\gamma_{q-2}<\gamma_{q-1}<f^{q}\left(\gamma_{0}\right)
$$

and we define

$$
\gamma^{\prime}=\bigvee_{i=0}^{q-1} f^{q-i}\left(\gamma_{i}\right)
$$

As in the proof of Lemma 8 we get $\gamma^{\prime}<f\left(\gamma^{\prime}\right)$. In particular, we obtain an essential circle $\gamma^{\prime}$ that is disjoint from its image under $f$.

To prove Lemma 10, we need the following lemma:

Lemma 11. Let $f \in \operatorname{Homeo}_{*}^{\wedge}(\mathbb{A})$ and $F$ be a lift of $f$ to $\mathbb{R}^{2}$. Suppose that there exist two path connected sets $X_{1}$ and $X_{2}$ in $\mathbb{R}^{2}$ satisfying

(1) $F\left(X_{i}\right) \cap X_{i}=\emptyset$ for $i=1,2$;

(2) $T^{k}\left(X_{i}\right) \cap X_{i}=\emptyset(i=1,2)$ for every $k \in \mathbb{Z} \backslash\{0\}$;

(3) either $X_{1}=X_{2}$ or $T^{k}\left(X_{1}\right) \cap X_{2}=\emptyset$ for every $k \in \mathbb{Z}$;

(4) there exist positive integers $p_{i}, q_{i}(i=1,2)$ such that

$$
F^{q_{1}}\left(X_{1}\right) \cap T^{p_{1}}\left(X_{1}\right) \neq \emptyset \quad \text { and } \quad F^{q_{2}}\left(X_{2}\right) \cap T^{-p_{2}}\left(X_{2}\right) \neq \emptyset .
$$

Then $F$ has a fixed point.

Proof. Since $F^{q_{1}}\left(X_{1}\right) \cap T^{p_{1}}\left(X_{1}\right) \neq \emptyset$ and $F^{q_{2}}\left(X_{2}\right) \cap T^{-p_{2}}\left(X_{2}\right) \neq \emptyset$, there exist points $x_{i}, x_{i}^{\prime} \in X_{i}(i=1,2)$ such that $F^{q_{1}}\left(x_{1}\right)=T^{p_{1}}\left(x_{1}^{\prime}\right)$ and $F^{q_{2}}\left(x_{2}\right)=T^{-p_{2}}\left(x_{2}^{\prime}\right)$. In the case where $X_{1} \neq X_{2}$, we choose a segment $\Gamma_{1}$ (resp. $\Gamma_{2}$ ) of $X_{1}$ (resp. $X_{2}$ ) that contains $x_{1}$ and $x_{1}^{\prime}$ (resp. $x_{2}$ and $x_{2}^{\prime}$ ). In the case where $X_{1}=X_{2}$, we choose a finite tree included in $X_{1}$ which contains $x_{i}, x_{i}^{\prime}(i=1,2)$ and we write $\Gamma_{1}=\Gamma_{2}$ for this tree. Then we can replace the couple $\left(X_{1}, X_{2}\right)$ by the couple $\left(\Gamma_{1}, \Gamma_{2}\right)$ which satisfies the same property. The items (2) and (3) of Lemma 11 imply that $\pi\left(\Gamma_{1}\right)$ and $\pi\left(\Gamma_{2}\right)$ are two disjoint segments or a finite tree in $\mathbb{A}$.

To prove by contradiction, we suppose that $F$ has no fixed point. We can find a maximal free brick decomposition $\mathfrak{B}$ of the plane that is $T$-invariant such that $B_{1}$ and $B_{2}$ are bricks of this decomposition and contain $\Gamma_{1}$ and $\Gamma_{2}$ respectively (note here that $B_{1}=B_{2}$ if $\left.X_{1}=X_{2}\right)$. We have that $B_{1} \prec T^{p_{1}}\left(B_{1}\right)$ and $B_{2} \prec T^{-p_{2}}\left(B_{2}\right)$ by the hypotheses where $B \prec B^{\prime}$ means $B \preceq B^{\prime}$ but $B^{\prime} \npreceq B$. This implies that, for every $n, n^{\prime} \geq 1$, we have

$$
B_{1} \prec T^{n p_{1}}\left(B_{1}\right) \quad \text { and } \quad B_{2} \prec T^{-n^{\prime} p_{2}}\left(B_{2}\right) .
$$

Now consider the sets

$$
\bigcup_{k \in \mathbb{Z}} T^{k}\left(B_{1 \succeq}\right)=\bigcup_{k \in \mathbb{Z}} T^{k}\left(B_{1}\right)_{\succeq} \text { and } \bigcup_{k \in \mathbb{Z}} T^{k}\left(B_{2 \succeq}\right)=\bigcup_{k \in \mathbb{Z}} T^{k}\left(B_{2}\right)_{\succeq} .
$$

We claim that the inclusions $B_{2} \subset \bigcup_{k \in \mathbb{Z}} T^{k}\left(B_{1 \succeq}\right)$ and $B_{1} \subset \bigcup_{k \in \mathbb{Z}} T^{k}\left(B_{2 \succeq}\right)$ cannot happen simultaneously. Otherwise, there exist integers $k$ and $k^{\prime}$ satisfying the fact that

$$
T^{k}\left(B_{1}\right) \preceq B_{2} \quad \text { and } \quad T^{k^{\prime}}\left(B_{2}\right) \preceq B_{1} .
$$

From (4.0.2) and (4.0.3), we get $T^{k+n^{\prime} p_{2}+k^{\prime}}\left(B_{1}\right) \prec B_{1}$ for every $n^{\prime} \geq 1$. When $n^{\prime}$ is large enough we have $k^{\prime \prime}=k+k^{\prime}+n^{\prime} p_{2}>0$. Hence, we have $T^{k^{\prime \prime}} p_{1}\left(B_{1}\right) \prec B_{1}$. By 
(4.0.2), we also have $B_{1} \prec T^{k^{\prime \prime}} p_{1}\left(B_{1}\right)$. It implies that $B_{1} \prec B_{1}$, which is impossible by the stronger version of Franks' Lemma. The fact that the inclusions $B_{2} \subset$ $\bigcup_{k \in \mathbb{Z}} T^{k}\left(B_{1 \succeq}\right)$ and $B_{1} \subset \bigcup_{k \in \mathbb{Z}} T^{k}\left(B_{2 \succeq}\right)$ cannot happen simultaneously implies that

(H1) : $\bigcup_{k \in \mathbb{Z}} T^{k}\left(B_{1 \succeq}\right) \cap \bigcup_{k \in \mathbb{Z}} T^{k}\left(B_{2} \preceq\right)=\emptyset \quad$ or $\quad(\mathrm{H} 2): \bigcup_{k \in \mathbb{Z}} T^{k}\left(B_{1} \preceq\right) \cap \bigcup_{k \in \mathbb{Z}} T^{k}\left(B_{2 \succeq}\right)=\emptyset$.

Suppose now that case (H1) happens. As $T^{p_{1}}\left(B_{1}\right) \in B_{1 \succeq}$ and $T^{p_{2}}\left(B_{2}\right) \in B_{2} \preceq$, we know that $\bigcup_{k \in \mathbb{Z}} T^{k}\left(B_{1 \succeq}\right)$ and $\bigcup_{k \in \mathbb{Z}} T^{k}\left(B_{2 \preceq}\right)$ are connected. This implies they project by $\pi$ onto essential connected surfaces (i.e. containing an essential circle) $b_{1} \succeq$ and $b_{2} \preceq$ with boundaries. So there exists at least a connected component of the boundary of $b_{1 \succeq}$ that is an essential circle and is free for $f$, which is contrary to the fact $f$ has the intersection property. We can also get a contradiction if case (H2) happens.

Hence, $F$ has at least one fixed point; we complete the proof.

Proof of Lemma 10. We know that the map $f$ satisfies the intersection property, that the lift $F \circ T^{q-1}$ is fixed point free, and that there is a positive recurrent point of $f$ with a positive rotation number for this new lift. By Theorem 5 , there is an oriented essential line $\gamma$ of $\mathbb{A}$ joining $S$ to $N$ that is lifted to an essential Brouwer line $\Gamma$ of $F \circ T^{q-1}$ which satisfies

$$
\Gamma<\left(F \circ T^{q-1}\right)(\Gamma) .
$$

We can write $\Gamma<T(\Gamma)<\left(F \circ T^{q}\right)(\Gamma)$. Write $F^{\prime}=F \circ T^{q}$; then we have

$$
F^{\prime-n}(\Gamma)<T^{-n}(\Gamma)<\Gamma<T^{n}(\Gamma)<F^{\prime n}(\Gamma) \text { for every } n \geq 1,
$$

which implies that $F^{\prime}$ is conjugate to a translation. We consider the annulus $\mathbb{A}^{\prime}=$ $\mathbb{R}^{2} / F^{\prime}$ and the homeomorphism $t$ induced by $T$ on $\mathbb{A}^{\prime}$. The map $F^{-1} \circ T^{q}=$ $F^{\prime-1} \circ T^{2 q}$ is a lift of $t^{2 q}$ that is fixed point free. By Theorem [5 there are three cases to consider:

(1) There exists an oriented essential line $\gamma^{\prime}$ of $\mathbb{A}^{\prime}$ that is lifted to an oriented line $\Gamma^{\prime}$ in $\mathbb{R}^{2}$ such that $\Gamma^{\prime}<F^{\prime}\left(\Gamma^{\prime}\right)$ and $\Gamma^{\prime}<\left(F^{-1} \circ T^{q}\right)\left(\Gamma^{\prime}\right)$.

(2) There exists an oriented essential line $\gamma^{\prime}$ of $\mathbb{A}^{\prime}$ that is lifted to an oriented line $\Gamma^{\prime}$ in $\mathbb{R}^{2}$ such that $\Gamma^{\prime}<F^{\prime}\left(\Gamma^{\prime}\right)$ and $\left(F^{-1} \circ T^{q}\right)\left(\Gamma^{\prime}\right)<\Gamma^{\prime}$.

(3) There exists an essential circle in $\mathbb{A}^{\prime}$ that is free for $t^{2 q}$.

We will get the lemma in the first case and contradictions in the two other cases.

In case (1), we have

$$
T^{-q}\left(\Gamma^{\prime}\right)<F\left(\Gamma^{\prime}\right)<T^{q}\left(\Gamma^{\prime}\right) .
$$

Applying $T^{q}$ to (4.0.4), we get $\Gamma^{\prime}<F^{\prime}\left(\Gamma^{\prime}\right)<T^{2 q}\left(\Gamma^{\prime}\right)$. Thus we have

$$
T^{-2 q n}\left(\Gamma^{\prime}\right)<F^{\prime-n}\left(\Gamma^{\prime}\right)<\Gamma^{\prime}<F^{\prime n}\left(\Gamma^{\prime}\right)<T^{2 q n}\left(\Gamma^{\prime}\right) \text { for every } n \geq 1 .
$$

Observe that $\bigcup_{n>1} R\left(F^{\prime-n}\left(\Gamma^{\prime}\right)\right) \cap L\left(F^{\prime n}\left(\Gamma^{\prime}\right)\right)=\mathbb{R}^{2}$, since $F^{\prime}$ is conjugate to a translation and the line $\gamma^{\prime}=\pi\left(\Gamma^{\prime}\right)$ is an essential line in $\mathbb{A}^{\prime}$. For any $P>0$, by the compactness of $[-q, q] \times[-P, P]$, there exists a positive integer $N$ such that $[-q, q] \times$ $[-P, P] \subset R\left(T^{-2 N q}\left(\Gamma^{\prime}\right)\right) \cap L\left(T^{2 N q}\left(\Gamma^{\prime}\right)\right)$. We deduce that $[-q,+\infty[\times[-P, P] \subset$ $R\left(T^{-2 N q}\left(\Gamma^{\prime}\right)\right)$ and $\left.]-\infty, q\right] \times[-P, P] \subset L\left(T^{2 N q}\left(\Gamma^{\prime}\right)\right)$, and hence

$$
\left[-q+2 N q,+\infty\left[\times[-P, P] \subset R\left(\Gamma^{\prime}\right)\right.\right.
$$


and

$$
]-\infty, q-2 N q\left[\times[-P, P] \subset L\left(\Gamma^{\prime}\right) .\right.
$$

This implies that $\Gamma^{\prime}$ is essential.

By Lemma 8, let $H_{1}=T, H_{2}=T^{q} \circ F^{-1}, H_{3}=T^{q} \circ F, q_{1}=2 q$ and $q_{2}=q_{3}=1$. We can construct an essential line $\Gamma^{\prime \prime}$ which satisfies

$$
T^{-q}\left(\Gamma^{\prime \prime}\right)<F\left(\Gamma^{\prime \prime}\right)<T^{q}\left(\Gamma^{\prime \prime}\right)
$$

and

$$
\Gamma^{\prime \prime}<T\left(\Gamma^{\prime \prime}\right)
$$

Hence, $\gamma^{\prime \prime}=\pi\left(\Gamma^{\prime \prime}\right)$ is an essential line of $\mathbb{A}$ that satisfies the conclusion of the lemma.

In case (2), we will see how to get a contradiction.

The line $\Gamma^{\prime}$ satisfies $\Gamma^{\prime}<\left(F \circ T^{q}\right)\left(\Gamma^{\prime}\right)$ and $\Gamma^{\prime}<\left(F \circ T^{-q}\right)\left(\Gamma^{\prime}\right)$. We define the set $X$ of couples of integers $(m, n)$ such that $\Gamma^{\prime}<\left(F^{m} \circ T^{n}\right)\left(\Gamma^{\prime}\right)$. It is a set stable by addition that contains $(1, q)$ and $(1,-q)$. So it contains all the integers $(m+n, q(m-n))$, where $m \geq 0, n \geq 0$ and at least one is non-zero. This is exactly the set of couples $(m, n q)$ where $m>0$ and $|n| \leq m$. For every couple $(m, n)$ of integers such that $m>0$ and $\left|\frac{n}{m}\right| \leq q-\frac{2}{m}$, we have $q m-1>0$ and $|n-1| \leq|n|+1 \leq q m-1$. Therefore, we have $q(m, n)-(1, q) \in X$, which means

$$
\Gamma^{\prime}<\left(F \circ T^{q}\right)\left(\Gamma^{\prime}\right)<\left(F^{m} \circ T^{n}\right)^{q}\left(\Gamma^{\prime}\right) .
$$

Fix $z \in \operatorname{Rec}^{+}(f)$ having a rotation number $\rho$ and $\widetilde{z} \in \pi^{-1}(z)$. Since $F^{\prime}=F \circ T^{q}$ is conjugate to a translation and the line $\gamma^{\prime}$ is an essential line in $\mathbb{A}^{\prime}$, we can always suppose that $\widetilde{z}$ is contained in the region $\mathrm{Cl}\left(R\left(\Gamma^{\prime}\right)\right) \cap L\left(\left(F \circ T^{q}\right)\left(\Gamma^{\prime}\right)\right)$ by replacing $\Gamma^{\prime}$ with an iterate $F^{\prime k}\left(\Gamma^{\prime}\right)$ if necessary. Consider the homeomorphism $f_{q}$ of the annulus $\mathbb{A}_{q}=\mathbb{R}^{2} / T^{q}$ lifted by $F$ and write $\pi_{q}: \mathbb{R}^{2} \rightarrow \mathbb{A}_{q}$ for the covering map. We know that $\operatorname{Rec}^{+}(f)=\operatorname{Rec}^{+}\left(f^{q}\right)$, and we can prove similarly that $\pi_{q}(\widetilde{z}) \in \operatorname{Rec}^{+}\left(f_{q}^{q}\right)$ (we give a proof in the Appendix; see Lemma 20). In other words, there exist two sequences of integers $\left(n_{i}\right)_{i \geq 1}$ and $\left(m_{i}\right)_{i \geq 1}$ such that $m_{i} \rightarrow+\infty$ and $F^{q m_{i}} \circ T^{q n_{i}}(\widetilde{z}) \rightarrow \widetilde{z}$ as $i \rightarrow+\infty$. Certainly, $\frac{q n_{i}}{q m_{i}} \rightarrow-\rho$ as $i \rightarrow+\infty$. Therefore, there is a positive integer $N$ such that when $i \geq N$, we have $\left|\frac{n_{i}}{m_{i}}\right|<q-1<q-\frac{2}{m_{i}}$.

By the inequation (4.0.6), we have $\Gamma^{\prime}<\left(F \circ T^{q}\right)\left(\Gamma^{\prime}\right)<\left(F^{m_{i}} \circ T^{n_{i}}\right)^{q}\left(\Gamma^{\prime}\right)$ when $i \geq N$. On one hand, the points of the sequence $\left\{\left(F^{m_{i}} \circ T^{n_{i}}\right)^{q}(\widetilde{z})\right\}_{i \geq N}$ belong to $R\left(F \circ T^{q}\left(\Gamma^{\prime}\right)\right)$, and so their limit belongs to $\mathrm{Cl}\left(R\left(F \circ T^{q}\left(\Gamma^{\prime}\right)\right)\right)$. On the other hand, the limit of the sequence belongs to $L\left(F \circ T^{q}\left(\Gamma^{\prime}\right)\right)$, which is a contradiction.

In case (3), we will get a contradiction again.

By Lemma 9, we deduce that there exists an essential circle $\gamma^{\prime}$ free for $t$. Therefore $\gamma^{\prime}$ lifts to a Brouwer line $\Gamma^{\prime}$ for $T$, in particular, $\Gamma^{\prime} \cap T\left(\Gamma^{\prime}\right)=\emptyset$.

The curve $\gamma^{\prime}$ is closed in $\mathbb{A}^{\prime}$, which implies that its lift $\Gamma^{\prime}$ satisfies

$$
\Gamma^{\prime}=F^{\prime}\left(\Gamma^{\prime}\right)=\left(F \circ T^{q}\right)\left(\Gamma^{\prime}\right) .
$$

Hence

$$
T^{-q}\left(\Gamma^{\prime}\right)=F\left(\Gamma^{\prime}\right) \quad \text { and } \quad F\left(\Gamma^{\prime}\right) \cap T^{1-q}\left(\Gamma^{\prime}\right)=\emptyset .
$$

Considering the map $F^{\prime \prime}=F \circ T^{q-1}$, we have the following:

- there exists a free line $\Gamma^{\prime}$ of $\mathbb{R}^{2}$ for $F^{\prime \prime}$ and for $T$ (and hence for $T^{k}$ for every $k \neq 0)$ such that $T^{-1}\left(\Gamma^{\prime}\right)=F^{\prime \prime}\left(\Gamma^{\prime}\right)$;

- $\emptyset \neq \operatorname{Rot}\left(F^{\prime \prime}\right) \subset[1,2 q-3]$; in particular, $F^{\prime \prime}$ has no fixed point. 
The first item above implies that there exists a segment $\Gamma_{0} \subset \Gamma^{\prime}$ such that $F^{\prime \prime}\left(\Gamma_{0}\right) \cap$ $T^{-1}\left(\Gamma_{0}\right) \neq \emptyset$ and that $\Gamma_{0}$ is free both for $F^{\prime \prime}$ and for $T^{k}$ for any $k \in \mathbb{Z} \backslash\{0\}$.

The second item above implies that there exists $z \in \mathbb{A}$ such that $z \in \operatorname{Rec}^{+}(f)$ and $\rho\left(F^{\prime \prime} ; z\right) \in[1,2 q-3]$. We claim that $z \notin \pi\left(\Gamma_{0}\right)$. Otherwise, choose a lift $\widetilde{z}$ of $z$ in $\Gamma_{0}$. Let $U$ be an open disk containing $\widetilde{z}$, located in the region between $T^{-1}\left(\Gamma^{\prime}\right)$ and $T\left(\Gamma^{\prime}\right)$. There exist positive integers $n \geq 2, l \geq 1$ such that $F^{\prime \prime n}(\widetilde{z}) \in T^{l}(U)$. As we have $F^{\prime \prime n}(\widetilde{z}) \in F^{\prime \prime n}\left(\Gamma_{0}\right) \subset T^{-n}\left(\Gamma^{\prime}\right)$, we deduce that $T^{l}(U) \cap T^{-n}\left(\Gamma^{\prime}\right) \neq \emptyset$, which is impossible. We have completed the claim. As $z \notin \pi\left(\Gamma_{0}\right)$, we can find a disk $U^{\prime}$ free for $F^{\prime \prime}$, containing $\widetilde{z}$, disjoint from $\Gamma_{0}$, and satisfying $T^{k}\left(U^{\prime}\right) \cap U^{\prime}=\emptyset$ for every $k \neq 0$ and $F^{\prime \prime n}\left(U^{\prime}\right) \cap T^{l}\left(U^{\prime}\right) \neq \emptyset$.

By Lemma 11, $F^{\prime \prime}$ has a fixed point, which is impossible.

In the rest of this section, we give some remarks about the relations between the positively recurrent set and the rotation number set.

Let $f \in \operatorname{Homeo}_{*}(\mathbb{A})$ and $F$ be a lift of $f$ to $\mathbb{R}^{2}$. Suppose that $z \in \operatorname{Rec}^{+}(f)$ and $\widetilde{z} \in \pi^{-1}(z)$. We define $\mathcal{E}(z) \subset \mathbb{R} \cup\{-\infty,+\infty\}$ by saying that $\rho \in \mathcal{E}(z)$ if there exists a sequence $\left\{n_{k}\right\}_{k=1}^{+\infty} \subset \mathbb{N}$ such that

- $\lim _{k \rightarrow+\infty} f^{n_{k}}(z)=z$;

- $\lim _{k \rightarrow+\infty} \frac{p_{1}\left(F^{n_{k}}(\widetilde{z})\right)-p_{1}(\widetilde{z})}{n_{k}}=\rho$.

Define $\rho^{-}(F ; z)=\inf \mathcal{E}(z)$ and $\rho^{+}(F ; z)=\sup \mathcal{E}(z)$. Obviously, we have that $\rho(F ; z)$ exists if and only if $\rho^{-}(F ; z)=\rho^{+}(F ; z) \in \mathbb{R}$.

The following proposition is due to Franks [F1] when $\mathbb{A}$ is a closed annulus and $f$ has no wandering point, and it was improved by Le Calvez Lec2] when $\mathbb{A}$ is an open annulus and $f$ satisfies the intersection property. We can use Lemma 11 to prove it.

Proposition 12. Let $f \in \operatorname{Homeo}_{*}^{\wedge}(\mathbb{A})$ and $F$ be a lift of $f$ to $\mathbb{R}^{2}$. Suppose that there exist two recurrent points $z_{1}$ and $z_{2}$ such that $-\infty \leq \rho^{-}\left(F ; z_{1}\right)<\rho^{+}\left(F ; z_{2}\right) \leq+\infty$. Then for any rational number $p / q \in] \rho^{-}\left(F ; z_{1}\right), \rho^{+}\left(F ; z_{2}\right)$ [ written in an irreducible way, there exists a periodic point of period $q$ whose rotation number is $p / q$.

Proof. We consider the map $f^{q}$ and its lift $F^{\prime}=F^{q} \circ T^{-p}$. It is sufficient to prove that $F^{\prime}$ has a fixed point.

By Lemma 9. $f^{q}$ satisfies the intersection property. By the properties of the rotation number, we have $\rho^{-}\left(F^{\prime} ; z_{1}\right)<0<\rho^{+}\left(F^{\prime} ; z_{2}\right)$. So we can choose open disks $b_{1}$ and $b_{2}$, containing respectively $z_{1}$ and $z_{2}$, equal if $z_{1}=z_{2}$, disjoint if $z_{1} \neq z_{2}$, such that the connected components of $\pi^{-1}\left(b_{1}\right)$ and $\pi^{-1}\left(b_{2}\right)$ are free for $F^{\prime}$. Choose a component $\widetilde{b}_{1}$ of $\pi^{-1}\left(b_{1}\right)$ and a component $\widetilde{b}_{2}$ of $\pi^{-1}\left(b_{2}\right)$ (equal to $\widetilde{b}_{1}$ if $z_{1}=z_{2}$ ). Observe that $\widetilde{b}_{1}$ and $\widetilde{b}_{2}$ satisfy all the hypotheses of Lemma 11] Then, Proposition 12 follows from Lemma 11

From Lemma 11] and Proposition 12, we have the following corollary:

Corollary 13. Let $f \in \operatorname{Homeo}_{*}^{\wedge}(\mathbb{A})$ and $F$ be a lift of $f$ to $\mathbb{R}^{2}$. If $\operatorname{Rec}^{+}(f) \neq \emptyset$, then $\operatorname{Rot}(F) \neq \emptyset$.

Proof. Let $z \in \operatorname{Rec}^{+}(f)$ and $\widetilde{z}$ be a lift of $z$. If $z$ is a fixed point, the corollary is true. Otherwise, choose a free disk $b$ for $f$ in $\mathbb{A}$ that contains $z$ and a connected component $\widetilde{b}$ of $\pi^{-1}(b)$. 
If $\rho^{-}(F ; z) \neq \rho^{+}(F ; z)$, then $\operatorname{Rot}(F) \neq \emptyset$ by Proposition [12, If $\rho^{-}(F ; z)=$ $\rho^{+}(F ; z) \in \mathbb{R}$, then $z$ has a rotation number and therefore $\operatorname{Rot}(F) \neq \emptyset$. It remains to study the cases $\rho^{-}(F ; z)=\rho^{+}(F ; z)=+\infty$ and $\rho^{-}(F ; z)=\rho^{+}(F ; z)=-\infty$. They are similar. We will now explain why the condition $\rho^{-}(F ; z)=\rho^{+}(F ; z)=+\infty$ implies that $f$ has a fixed point and therefore that $\operatorname{Rot}(F) \neq \emptyset$. We can find two rational numbers $\frac{p_{i}}{q_{i}}(i=1,2)$ and an integer $n$, arbitrarily large, such that $p_{1} / q_{1}<n<p_{2} / q_{2}, F^{q_{1}}(\widetilde{b}) \cap T^{p_{1}}(\widetilde{b}) \neq \emptyset$ and $F^{q_{2}}(\widetilde{b}) \cap T^{p_{2}}(\widetilde{b}) \neq \emptyset$. Consider the lift $F^{\prime}=F \circ T^{-n}$ of $f$ to $\mathbb{R}^{2}$; we have $F^{\prime q_{1}}(\widetilde{b}) \cap T^{p_{1}-q_{1} n}(\widetilde{b}) \neq \emptyset$ and $F^{\prime q_{2}}(\widetilde{b}) \cap T^{p_{2}-q_{2} n}(\widetilde{b}) \neq$ $\emptyset$. Recall that $\widetilde{b}$ is free for $F^{\prime}$. By Lemma 11, we have that $F^{\prime}$ has a fixed point and hence $f$ has a fixed point. We have completed the proof.

Remark 2. Let $f \in \operatorname{Homeo}_{*}^{\wedge}(\mathbb{A})$ and $F$ be a lift of $f$ to $\mathbb{R}^{2}$. Obviously, if the set $\operatorname{Rot}(F)$ is reduced to a single irrational number $\rho$, then $\operatorname{Rec}^{+}(f) \neq \emptyset$ and $f$ has no periodic orbit. Conversely, if $\operatorname{Rec}^{+}(f) \neq \emptyset$ and $f$ has no periodic orbit, by Proposition 12 and Corollary [13, it is easy to prove that $\operatorname{Rot}(F)$ is reduced to a single number $\rho$. But it is not so clear that the number $\rho$ should be irrational. However, the number $\rho$ would be irrational if $f$ further preserves a finite measure whose support is $\mathbb{A}$, which was proved in Proposition 0.2 of $\mathrm{BCL}$.

\section{WEAK ROTATION NUMBER AND THE LINE TRANSLATION THEOREM}

We have supposed previously that there exists a recurrent point. It is natural to wonder if the main theorem is still true without this hypothesis.

In this section, we define the weak rotation set of $f$ which is a generalization of the rotation set defined in Section 1. It was introduced in [F2] in a more generalized framework (see also Ler2 for a local study). We first give some properties of weak rotation numbers, then we prove the generalization of the line translation theorem.

Fix $f \in \operatorname{Homeo}_{*}^{\wedge}(\mathbb{A})$ and a lift $F$. For every compact set $K$ define the set $\operatorname{Rot}_{\text {weak }, K}(F)$ in the following way: $r \in[-\infty,+\infty]$ belongs to $\operatorname{Rot}_{\text {weak }, K}(F)$ if there exists a sequence $\left\{\widetilde{z}_{k}\right\}_{k \geq 1} \subset \mathbb{R}^{2}$ and a sequence $\left\{n_{k}\right\}_{k \geq 1}$ of positive integers such that

- $\pi\left(\widetilde{z}_{k}\right) \in K$;

- $\pi\left(F^{n_{k}}\left(\widetilde{z}_{k}\right)\right) \in K$;

- $\lim _{k \rightarrow+\infty} n_{k}=+\infty$;

- $\lim _{k \rightarrow+\infty} \frac{p_{1}\left(F^{n_{k}}\left(\widetilde{z}_{k}\right)\right)-p_{1}\left(\widetilde{z}_{k}\right)}{n_{k}}=r$.

Define the weak rotation set of $F$ as

$$
\operatorname{Rot}_{\text {weak }}(F)=\bigcup_{K \in C(\mathbb{A})} \operatorname{Rot}_{\text {weak }, K}(F),
$$

where $C(\mathbb{A})$ is the collection of all compact subsets of $\mathbb{A}$.

Proposition 14. If $f \in \operatorname{Homeo}_{*}^{\wedge}(\mathbb{A})$, we have the following properties:

(1) $\operatorname{Rot}_{\text {weak }, K}(F)$ is closed.

(2) If $K$ separates the two ends $N$ and $S$ of $\mathbb{A}$, then $\operatorname{Rot}_{\text {weak }, K}(F) \neq \emptyset$. Therefore $\operatorname{Rot}_{\text {weak }}(F) \neq \emptyset$.

(3) $\operatorname{Rot}(F) \subset \operatorname{Rot}_{\text {weak }}(F)$.

(4) For every $p \in \mathbb{Z}$ and $q \in \mathbb{Z}$, we have $\operatorname{Rot}_{\text {weak }}\left(F^{q} \circ T^{p}\right)=q \operatorname{Rot}_{\text {weak }}(F)+p$. 
Proof. (1) For every $n \geq 1$ define a set $R_{n} \subset \mathbb{R}$ as

$$
R_{n}=\left\{\frac{p_{1} \circ F^{n}(\widetilde{z})-p_{1}(\widetilde{z})}{n} \mid \widetilde{z} \in \pi^{-1}(K) \quad \text { and } \quad F^{n}(\widetilde{z}) \in \pi^{-1}(K)\right\} .
$$

Observe now that

$$
\operatorname{Rot}_{\text {weak }, K}(F)=\bigcap_{n \geq 1} \mathrm{Cl}\left(\bigcup_{k \geq n} R_{k}\right),
$$

so $\operatorname{Rot}_{\text {weak, } K}(F)$ is closed.

(2) To prove that $\operatorname{Rot}_{\text {weak }, K}(F)$ is not empty, it is sufficient to prove that for every $q \geq 1$, there is a point $z_{q} \in K$ such that $f^{q}\left(z_{q}\right) \in K$. Suppose that there exists $q \geq 1$ such that $K \cap f^{q}(K)=\emptyset$. As $K$ is compact, we can find an open neighborhood $U$ of $K$ such that $U \cap f^{q}(U)=\emptyset$. Since $K$ separates the two ends of $\mathbb{A}$, we deduce that $U$ contains an essential circle. This contradicts Lemma 9

(3) For every $r \in \operatorname{Rot}(F)$, there exists $z \in \operatorname{Rec}^{+}(f)$ such that $\rho(F ; z)=r$. We choose $K$ to be a closed disk whose interior contains $z$. By the definitions, we have $r=\rho(F ; z) \in \operatorname{Rot}_{\text {weak }, K}(F) \subset \operatorname{Rot}_{\text {weak }}(F)$. Therefore, $\operatorname{Rot}(F) \subset \operatorname{Rot}_{\text {weak }}(F)$.

(4) Since $F \circ T=T \circ F$, we clearly have $\operatorname{Rot}_{\text {weak }}\left(F \circ T^{p}\right)=\operatorname{Rot}_{\text {weak }}(F)+p$. Let us now prove that $\operatorname{Rot}_{\text {weak }}\left(F^{q}\right)=q \operatorname{Rot}_{\text {weak }}(F)$ for every $q \in \mathbb{Z}$. When $q=0$, it is trivial. First, fix $q>0$. Obviously, $\operatorname{Rot}_{\text {weak }, K}\left(F^{q}\right) \subset q \operatorname{Rot}_{\text {weak }, K}(F)$ for any $K \in \mathrm{C}(\mathbb{A})$ by definition. Therefore, $\operatorname{Rot}_{\text {weak }}\left(F^{q}\right) \subset q \operatorname{Rot}_{\text {weak }}(F)$.

Now, we prove the inverse. For every $r \in \operatorname{Rot}_{\text {weak }}(F)$, there exists $K \in \mathrm{C}(\mathbb{A})$ such that $r \in \operatorname{Rot}_{\text {weak }, K}(F)$. Therefore, there exists a sequence $\left\{\widetilde{z}_{k}\right\}_{k \geq 1} \subset \mathbb{R}^{2}$ and a sequence $\left\{n_{k}\right\}_{k \geq 1}$ of positive integers such that

- $\pi\left(\widetilde{z}_{k}\right) \in K$;

- $\pi\left(F^{n_{k}}\left(\widetilde{z}_{k}\right)\right) \in K$

- $\lim _{k \rightarrow+\infty} n_{k}=+\infty$;

- $\lim _{k \rightarrow+\infty} \frac{p_{1}\left(F^{n_{k}}\left(\widetilde{z}_{k}\right)\right)-p_{1}\left(\widetilde{z}_{k}\right)}{n_{k}}=r$.

Write $n_{k}=l_{k} q+p_{k}$ where $0 \leq p_{k}<q$. Suppose that there are infinitely many $k$ such that $p_{k}=p$ where $0 \leq p<q$. We can choose a subsequence $\left\{n_{k_{j}}\right\}_{j \geq 1}$ of $\left\{n_{k}\right\}_{k \geq 1}$ such that $n_{k_{j}}=l_{k_{j}} q+p$ and

$$
\lim _{j \rightarrow+\infty} \frac{p_{1}\left(F^{q l_{k_{j}}}\left(F^{p}\left(\widetilde{z}_{k_{j}}\right)\right)\right)-p_{1}\left(F^{p}\left(\widetilde{z}_{k_{j}}\right)\right)}{q l_{k_{j}}}=r .
$$

Then we have

$$
q r=\lim _{j \rightarrow+\infty} \frac{p_{1}\left(F^{q l_{k_{j}}}\left(F^{p}\left(\widetilde{z}_{k_{j}}\right)\right)\right)-p_{1}\left(F^{p}\left(\widetilde{z}_{k_{j}}\right)\right)}{l_{k_{j}}} \in \operatorname{Rot}_{\text {weak }, f^{p}(K)}\left(F^{q}\right) .
$$

Hence $q \operatorname{Rot}_{\text {weak }}(F) \subset \operatorname{Rot}_{\text {weak }}\left(F^{q}\right)$.

For the case $q<0$, it is sufficient to prove that $\operatorname{Rot}_{\text {weak }, K}\left(F^{-1}\right)=-\operatorname{Rot}_{\text {weak }, K}(F)$ for every $K \in \mathrm{C}(\mathbb{A})$. For every $r \in \operatorname{Rot}_{\text {weak }, K}(F)$, by letting $\widetilde{z}_{k}^{\prime}=F^{n_{k}}\left(\widetilde{z}_{k}\right)$ in the definition of $\operatorname{Rot}_{\text {weak }, K}(F)$, we have

- $\pi\left(\widetilde{z}_{k}^{\prime}\right) \in K$;

- $\pi\left(F^{-n_{k}}\left(\widetilde{z}_{k}^{\prime}\right)\right) \in K$;

- $\lim _{k \rightarrow+\infty} n_{k}=+\infty$;

- $\lim _{k \rightarrow+\infty} \frac{p_{1}\left(F^{-n_{k}}\left(\widetilde{z}_{k}^{\prime}\right)\right)-p_{1}\left(\widetilde{z}_{k}^{\prime}\right)}{n_{k}}=-r$. 
Therefore,

$$
-\operatorname{Rot}_{\text {weak }, K}(F) \subset \operatorname{Rot}_{\text {weak }, K}\left(F^{-1}\right) .
$$

By replacing $F$ by $F^{-1}$ in conclusion (5.0.7), we have

$$
\operatorname{Rot}_{\text {weak }, K}\left(F^{-1}\right) \subset-\operatorname{Rot}_{\text {weak }, K}(F) .
$$

We have completed the proof.

Lemma 15. Suppose that there exists a compact set $K \subset \mathbb{A}$ and an oriented line $\Gamma$ of $\mathbb{R}^{2}$ satisfying

(1) $\Gamma<T(\Gamma)$;

(2) $\Gamma<F(\Gamma)$ (resp. $F(\Gamma)<\Gamma)$;

(3) $\pi^{-1}(K) \subset \bigcup_{k \in \mathbb{Z}} T^{k}(\mathrm{Cl}(R(\Gamma)) \cap L(T(\Gamma)))$.

Then $\operatorname{Rot}_{\text {weak }, K}(F) \subset[0,+\infty]$ (resp. $\left.\operatorname{Rot}_{\text {weak }, K}(F) \subset[-\infty, 0]\right)$. As a consequence, if $\Gamma$ is a lift of an oriented essential line in $\mathbb{A}$ joining $S$ to $N$, satisfying $\Gamma<F(\Gamma)$ (resp. $F(\Gamma)<\Gamma)$, then $\operatorname{Rot}_{\text {weak }}(F) \subset[0,+\infty]\left(\right.$ resp. $\left.\operatorname{Rot}_{\text {weak }}(F) \subset[-\infty, 0]\right)$.

Proof. We proceed by contradiction. We suppose that $\Gamma<F(\Gamma)$ and that there exists a negative number $\rho$ such that $\rho \in \operatorname{Rot}_{\text {weak, } K}(F)$. Write

$$
\widetilde{K}=\pi^{-1}(K) \cap \mathrm{Cl}(R(\Gamma)) \cap L(T(\Gamma))
$$

and

$$
\operatorname{width}(\widetilde{K})=\sup _{\widetilde{z}_{1}, \widetilde{z}_{2} \in \widetilde{K}}\left\{p_{1}\left(\widetilde{z}_{1}\right)-p_{1}\left(\widetilde{z}_{2}\right)\right\} .
$$

We first claim that $\operatorname{width}(\widetilde{K})$ is finite. According to hypothesis (3), we have $K=\pi(\widetilde{K})$. For every $k \geq 1$, we define an open set

$$
\widetilde{U}_{k}=\left(R\left(T^{-1}(\Gamma)\right) \cap L(T(\Gamma))\right) \cap\left\{(x, y) \in \mathbb{R}^{2} \mid-k<x<k\right\} .
$$

By hypothesis (1), the sequence of open sets $\left\{\pi\left(\widetilde{U}_{k}\right)\right\}_{k>1}$ in $\mathbb{A}$ is increasing. Since $\mathrm{Cl}(R(\Gamma)) \cap L(T(\Gamma)) \subset R\left(T^{-1}(\Gamma)\right) \cap L(T(\Gamma))$, we have $\widetilde{\widetilde{K}} \cap\left\{(x, y) \in \mathbb{R}^{2} \mid-k<x<\right.$ $k\} \subset \widetilde{U}_{k}$. Obviously, $\widetilde{K} \subset \bigcup_{k>1} \widetilde{U}_{k}$ and $K \subset \bigcup_{k \geq 1} \pi\left(\widetilde{U}_{k}\right)$. As $K$ is compact, there is a positive integer $N$ such that $K \subset \pi\left(\widetilde{U}_{N}\right)$. It implies that, for every $\widetilde{z} \in \widetilde{K}$, there exists $k \in \mathbb{Z}$ such that $T^{k}(\widetilde{z}) \in \widetilde{U}_{N}$. Observe that

$$
T^{l}\left(R\left(T^{-1}(\Gamma)\right) \cap L(T(\Gamma))\right) \cap(\mathrm{Cl}(R(\Gamma)) \cap L(T(\Gamma)))=\emptyset \quad \text { if } \quad l \in \mathbb{Z} \backslash\{0,1\} .
$$

It implies that the only possibilities for $k$ are -1 and 0 . So $\widetilde{K} \subset \widetilde{U}_{N} \cup T\left(\widetilde{U}_{N}\right)$. It completes the claim.

By the definition of $\operatorname{Rot}_{\text {weak }, K}(F)$, there is a sequence $\left\{\widetilde{z}_{k}\right\}_{k \geq 1} \subset \widetilde{K}$ and a sequence $\left\{n_{k}\right\}_{k \geq 1}$ of positive integers such that

- $\lim _{k \rightarrow+\infty} n_{k}=+\infty$;

- $\left\{\pi\left(F^{n_{k}}\left(\widetilde{z}_{k}\right)\right)\right\}_{k \geq 1} \subset K$;

- $\lim _{k \rightarrow+\infty} \frac{p_{1}\left(F^{n_{k}}\left(\widetilde{z}_{k}\right)\right)-p_{1}\left(\widetilde{z}_{k}\right)}{n_{k}}=\rho$.

If $k$ is large enough, then

$$
p_{1}\left(F^{n_{k}}\left(\widetilde{z}_{k}\right)\right)-p_{1}\left(\widetilde{z}_{k}\right)<-2 \operatorname{width}(\widetilde{K}) .
$$

It implies that $F^{n_{k}}\left(\widetilde{z}_{k}\right)$ is on the left of $\Gamma$. But, by hypothesis (2), for every $n \geq 1$ and $\widetilde{z} \in \widetilde{K}, F^{n}(\widetilde{z})$ is on the right of $\Gamma$; thus we get a contradiction. 
Let us now prove the second statement of the lemma. The line $\Gamma$ is a lift of an oriented essential line in $\mathbb{A}$ joining $S$ to $N$. Observe that

$$
\bigcup_{k \in \mathbb{Z}} T^{k}(\mathrm{Cl}(R(\Gamma)) \cap L(T(\Gamma)))=\mathbb{R}^{2}
$$

then we get the last consequence.

Using Lemma 15. we can get more information on the weak rotation number set of $F$.

Proposition 16. We have the following properties:

(1) If $\frac{p}{q}$ is given and if $\operatorname{Rot}_{\text {weak }, K}(F)$ contains $r_{1}$ and $r_{2}$ where $r_{1}<\frac{p}{q}<r_{2}$, then there exists $\widetilde{z}$ such that $F^{q}(\widetilde{z})=T^{p}(\widetilde{z})$.

(2) The sets $\mathrm{Cl}\left(\operatorname{Rot}_{\text {weak }}(F)\right)$ and $\mathrm{Cl}(\operatorname{Rot}(F))$ are the same intervals except in the case where $\operatorname{Rec}^{+}(f)=\emptyset$ and $\operatorname{Rot}_{\text {weak }}(F)$ is reduced to an element of $\mathbb{R}$.

Proof. To prove (1), consider the map $f^{q}$ and its lift $F^{\prime}=F^{q} \circ T^{-p}$. We must prove that $F^{\prime}$ has a fixed point. We suppose that $F^{\prime}$ has no fixed point. By Lemma 9. $f^{q}$ satisfies the intersection property. By Theorem 5, there exists an essential line of $\mathbb{A}$ joining $S$ to $N$ that lifts to a Brouwer line $\Gamma$ of $F^{\prime}$. Then either $\Gamma<F^{\prime}(\Gamma)$ or $F^{\prime}(\Gamma)<\Gamma$. By Lemma 15, we have either $\operatorname{Rot}_{\text {weak }}(F) \subset[0,+\infty]$ or $\operatorname{Rot}_{\text {weak }}(F) \subset[-\infty, 0]$. However, by assertion (4) of Proposition [14, we have $q r_{1}-p \in \operatorname{Rot}_{\text {weak }, K}\left(F^{\prime}\right)$ and $q r_{2}-p \in \operatorname{Rot}_{\text {weak, } K}\left(F^{\prime}\right)$ with $q r_{1}-p<0<q r_{2}-p$, which is impossible. Therefore, $F^{\prime}$ has a fixed point.

By assertion (3) of Proposition 14, we know that the inclusion $\mathrm{Cl}(\operatorname{Rot}(F)) \subset$ $\mathrm{Cl}\left(\operatorname{Rot}_{\text {weak }}(F)\right)$ is true. If $\operatorname{Rot}_{\text {weak }}(F)$ is reduced to a real number and if $\operatorname{Rec}^{+}(f) \neq$ $\emptyset$, then by Corollary 13, $\operatorname{Rot}(F)$ is not empty and therefore is equal to $\operatorname{Rot}_{\text {weak }}(F)$. To get (2), it is sufficient first to prove that $\mathrm{Cl}\left(\operatorname{Rot}_{\text {weak }}(F)\right) \subset \mathrm{Cl}(\operatorname{Rot}(F))$ in the case where $\operatorname{Rot}_{\text {weak }}(F)$ is not reduced to a point and then to explain why $\operatorname{Rot}_{\text {weak }}(F)$ cannot be reduced to $\{+\infty\}$ or $\{-\infty\}$.

Suppose that $\operatorname{Rot}_{\text {weak }}(F)$ is not reduced to a point and denote by $I$ the interior of its convex hull. According to (1), we have

$$
\mathrm{Cl}\left(\operatorname{Rot}_{\text {weak }}(F)\right) \subset \mathrm{Cl}(I)=\mathrm{Cl}(I \cap \mathbb{Q}) \subset \mathrm{Cl}\left(\operatorname{Rot}_{\text {per }}(F)\right) \subset \mathrm{Cl}(\operatorname{Rot}(F)),
$$

where $\operatorname{Rot}_{\text {per }}(F)$ is the set of rotation numbers of periodic points.

Now we turn to explain why $\operatorname{Rot}_{\text {weak }}(F)$ cannot be reduced to $\{+\infty\}$ or $\{-\infty\}$.

We state the first case; the other case is similar. Consider the sphere $\mathbf{S}^{2}=$ $\mathbb{A} \sqcup\{N, S\}$. Suppose first that there exists a non-trivial invariant continuum $K$ that contains the end $N$ but not the end $S$. Consider the connected component $U$ of $\mathbf{S}^{2} \backslash K$ that contains $S$. It is simply connected (a fact from plane topology) and invariant by $f$. We can define the prime end compactification of $U$, introduced by Carathéodory [C], by adding a circle $\mathbf{S}^{1}$. A very good exposition of the theory of prime ends in modern terminology can be found in the paper [M] of Mather.

The prime end compactification can be defined purely topologically but has another significance if we put a complex structure on $\mathbf{S}^{2}$. We can find a conformal map $\phi$ between $U$ and the open disk $\mathbb{D}=\{z \in \mathbb{C}|| z \mid<1\}$, and we put on $U \sqcup \mathbf{S}^{1}$ the topology (up to homeomorphism of the resulting space, is independent of $\phi$ ) induced from the natural topology of $\mathrm{Cl}(\mathbb{D})$ in $\mathbb{C}$ by the bijection

$$
\bar{\phi}: U \sqcup \mathbf{S}^{1} \rightarrow \mathrm{Cl}(\mathbb{D})
$$

equal to $\phi$ on $U$ and to the identity on $\mathbf{S}^{1}$. 
As $U$ is invariant by $f$, the map $\left.f\right|_{U}$ can be extended to a homeomorphism $\bar{f}$ of $U \sqcup \mathbf{S}^{1}$. Moreover, $\bar{f}$ restricted to the prime ends set $\mathbf{S}^{1}$ is an orientation preserving homeomorphism of the circle. Therefore, we can define its rotation number, say $\rho \in \mathbb{R} / \mathbb{Z}$.

We take off $S$ from $U \sqcup \mathbf{S}^{1}$ and then paste the annulus $\mathbf{S}^{1} \times[0,+\infty)$ and $U \sqcup \mathbf{S}^{1} \backslash\{S\}$ together along $\mathbf{S}^{1}$. We now define $f^{\prime}$ as the extension of the map $\bar{f}$ to the new open annulus $\mathbb{A}^{\prime}$ as follows:

$$
f^{\prime}(x, y)= \begin{cases}\bar{f}(x, y) & \text { if } \quad(x, y) \in U \sqcup \mathbf{S}^{1} \backslash\{S\}, \\ (g(x), y) & \text { if } \quad(x, y) \in \mathbf{S}^{1} \times[0,+\infty),\end{cases}
$$

where $g$ is the map induced by $\bar{f}$ on $\mathbf{S}^{1}$. By the construction of $f^{\prime}$, the map $f^{\prime}$ satisfies the intersection property. Indeed, we suppose that $\gamma$ is an essential circle in $\mathbb{A}^{\prime}$. If $\gamma$ does not meet $\mathbf{S}^{1} \times[0,+\infty)$, it must meet its image because $f$ satisfies the intersection property. Suppose now that $\gamma$ meets $\mathbf{S}^{1} \times[0,+\infty)$. There exists a real number $r \geq 0$ such that $\gamma$ meets $\mathbf{S}^{1} \times\{r\}$ but does not meet $\mathbf{S}^{1} \times(r,+\infty)$. By the definition of $f^{\prime}$, we have that $f^{\prime}(\gamma)$ does not meet $\mathbf{S}^{1} \times(r,+\infty)$. Without loss of generality, we suppose that $\gamma$ is not the curve $\mathbf{S}^{1} \times\{r\}$. As $\gamma$ is essential, there are exactly two unbounded connected components of $\mathbb{A}^{\prime} \backslash\left(\mathbf{S}^{1} \times\{r\} \cup \gamma\right)$. Let $C$ be the union of all bounded connected components of $\mathbb{A}^{\prime} \backslash\left(\mathbf{S}^{1} \times\{r\} \cup \gamma\right)$. Observing that $f^{\prime}(\gamma) \cap \mathbf{S}^{1} \times\{r\} \neq \emptyset$ and $C$ cannot contain any essential curve, we deduce that $f^{\prime}(\gamma)$ meets $\gamma$.

For any lift $F^{\prime}$ of $f^{\prime}$ to the universal cover of $\mathbb{A}^{\prime}$, we have the following facts:

- there exists $\rho \in \mathbb{R}$ such that $\operatorname{Rot}_{\text {weak, } \mathbf{S}^{1} \times\{r\}}\left(F^{\prime}\right)=\{\rho\}$ for every $r \geq 0$;

- $\operatorname{Rot}_{\text {weak }, K^{\prime}}\left(F^{\prime}\right)=\{+\infty\}$ if the set $K^{\prime}$ is a compact set of $U \backslash\{S\}$ that contains an essential circle.

So the set $\operatorname{Rot}_{\text {weak }}\left(F^{\prime}\right)$ is not reduced to a point. Assertion (1) implies that there are many periodic points with a rotation number different from $\rho$. They correspond to periodic points of our initial map $f$. We have a contradiction.

This implies that there exists a neighborhood of $N$ that is an isolating Jordan domain (see Section 2.4). Of course, we have a similar situation for $S$. Observe that there are no periodic orbits except $N$ and $S$ and that the intersection property guarantees that $N$ and $S$ are neither sinks nor sources. Thanks to Theorem 7 there exists an integer $k$ such that the Lefschetz indices $i\left(f^{k}, S\right)$ and $i\left(f^{k}, N\right)$ are non-positive. But because of the Lefschetz formula, we know that the sum is 2 (the Euler characteristic of $\mathbf{S}^{2}$ ). We have a contradiction.

Observe that the proofs of Lemma 8 and Lemma 9 do not use the existence of recurrent points. To adapt the line translation theorem with our new hypothesis, we only need to prove a result similar to Lemma 10.

Lemma 17. Let $f \in \operatorname{Homeo}_{*}^{\wedge}(\mathbb{A})$. We suppose that $F$ is a lift of $f$ to $\mathbb{R}^{2}$ and that

$$
\operatorname{Rot}_{\text {weak }}(F) \subset[-q+2, q-2],
$$

where $q \geq 2$. Then there exists an oriented essential line $\gamma$ in $\mathbb{A}$ joining $S$ to $N$ that is lifted to an essential line $\Gamma$ in $\mathbb{R}^{2}$ which satisfies

$$
T^{-q}(\Gamma)<F(\Gamma)<T^{q}(\Gamma) .
$$

Proof. According to assertion (2) of Proposition[16] and Lemma 10, we only need to consider the case where $\operatorname{Rec}^{+}(f)=\emptyset$ and $\operatorname{Rot}_{\text {weak }}(F)$ is reduced to a real number $\rho$. 
By assertion (4) of Proposition [14] we have $\operatorname{Rot}_{\text {weak }}\left(F \circ T^{q-1}\right)=\{\rho+q-1\} \subset$ $[1,2 q-3]$. Of course, the map $F \circ T^{q-1}$ has no fixed point. We choose a compact set $K$ such that $\operatorname{Rot}_{\text {weak }, K}\left(F \circ T^{q-1}\right)=\{\rho+q-1\}$. Note that the map $f$ satisfies the intersection property. By Theorem [5] and Lemma [15, there is an oriented essential line $\gamma$ of $\mathbb{A}$ joining $S$ to $N$ that is lifted to an essential Brouwer line $\Gamma$ of $F \circ T^{q-1}$ which satisfies

$$
\Gamma<\left(F \circ T^{q-1}\right)(\Gamma) .
$$

As in the proof of Lemma 10, we deduce that $F^{\prime}=F \circ T^{q}$ is conjugate to a translation. Here again, we consider the annulus $\mathbb{A}^{\prime}=\mathbb{R}^{2} / F^{\prime}$, the homeomorphism $t$ induced by $T$ on $\mathbb{A}^{\prime}$ and the lift $F^{-1} \circ T^{q}=F^{\prime-1} \circ T^{2 q}$ of $t^{2 q}$. As it is fixed point free, here again Theorem 5 asserts that there are three possible cases:

(1) There exists an oriented essential line $\gamma^{\prime}$ of $\mathbb{A}^{\prime}$ that is lifted to an oriented line $\Gamma^{\prime}$ in $\mathbb{R}^{2}$ such that $\Gamma^{\prime}<F^{\prime}\left(\Gamma^{\prime}\right)$ and $\Gamma^{\prime}<\left(F^{-1} \circ T^{q}\right)\left(\Gamma^{\prime}\right)$.

(2) There exists an oriented essential line $\gamma^{\prime}$ of $\mathbb{A}^{\prime}$ that is lifted to an oriented line $\Gamma^{\prime}$ in $\mathbb{R}^{2}$ such that $\Gamma^{\prime}<F^{\prime}\left(\Gamma^{\prime}\right)$ and $\left(F^{-1} \circ T^{q}\right)\left(\Gamma^{\prime}\right)<\Gamma^{\prime}$.

(3) There exists an essential circle in $\mathbb{A}^{\prime}$ that is free for $t^{2 q}$.

In case (1), the arguments that we gave in the proof of Lemma 10 permit us to construct an essential line of $\mathbb{A}$ that satisfies the conclusion of the lemma.

In case (2), we will show how to get a contradiction.

The line $\Gamma^{\prime}$ satisfies $\Gamma^{\prime}<\left(F \circ T^{q}\right)\left(\Gamma^{\prime}\right)$ and $\Gamma^{\prime}<\left(F \circ T^{-q}\right)\left(\Gamma^{\prime}\right)$. Here again, the set $X$ of the couple of integers $(m, n)$ such that $\Gamma^{\prime}<\left(F^{m} \circ T^{n}\right)\left(\Gamma^{\prime}\right)$ contains the set of couples $(m, n q)$, where $m>0$ and $|n| \leq m$ (see the proof of Lemma 10). Let $p$ be any given positive integer. For every couple $(m, n)$ of integers such that $m>\frac{p}{q}$ and $\left|\frac{n}{m}\right| \leq q-\frac{2 p}{m}$, we have $q m-p>0$ and $|n-p| \leq|n|+p \leq q m-p$. Therefore, we have $q(m, n)-p(1, q) \in X$, which means

$$
\Gamma^{\prime}<\left(F \circ T^{q}\right)^{p}\left(\Gamma^{\prime}\right)<\left(F^{m} \circ T^{n}\right)^{q}\left(\Gamma^{\prime}\right) .
$$

Let $\widetilde{K}_{q}=\pi^{-1}\left(\bigcup_{0<i<q} f^{i}(K)\right) \cap \operatorname{Cl}\left(R\left(T^{-q}(\Gamma)\right) \cap L(\Gamma)\right)$. Since $F^{\prime}=F \circ T^{q}$ is conjugate to a translation and $\gamma^{\prime}$ is an essential line in $\mathbb{A}^{\prime}$, we can always suppose that the compact set $\widetilde{K}_{q}$ is contained in the region $\mathrm{Cl}\left(R\left(\Gamma^{\prime}\right)\right) \cap L\left(\left(F \circ T^{q}\right)^{p}\left(\Gamma^{\prime}\right)\right)$ for some positive integer $p$ by replacing $\Gamma^{\prime}$ with an iterate $F^{\prime k}\left(\Gamma^{\prime}\right)$ if necessary. Consider the homeomorphism $f_{q}$ of the annulus $\mathbb{A}_{q}=\mathbb{R}^{2} / T^{q}$ lifted by $F$ and write $\pi_{q}: \mathbb{R}^{2} \rightarrow \mathbb{A}_{q}$ for the covering map. Similar to the proof of assertion (4) of Proposition 14, we can find a sequence $\left\{\widetilde{z}_{k}\right\}_{k \geq 1} \subset \widetilde{K}_{q}$, a sequence $\left\{m_{k}\right\}_{k \geq 1}$ of positive integers and a sequence $\left\{n_{k}\right\}_{k \geq 1}$ of integers such that

- $\lim _{k \rightarrow+\infty} m_{k}=+\infty$;

- $F^{q m_{k}}\left(\widetilde{z}_{k}\right) \in T^{-q n_{k}}\left(\widetilde{K}_{q}\right)$ for every $k \geq 1$;

- $\left.\frac{-q n_{k}}{q m_{k}} \in\right]-q+1, q-1[$ when $k$ is large enough.

Therefore, there is a positive integer $N$ such that when $k \geq N$, we have $m_{k}>$ $2 p>\frac{p}{q}$ and $\left|\frac{n_{k}}{m_{k}}\right|<q-1<q-\frac{2 p}{m_{k}}$.

By the inequation (5.0.8), we have $\Gamma^{\prime}<\left(F \circ T^{q}\right)^{p}\left(\Gamma^{\prime}\right)<\left(F^{m_{k}} \circ T^{n_{k}}\right)^{q}\left(\Gamma^{\prime}\right)$ when $k \geq N$. On the one hand, the points of the sequence $\left\{\left(F^{m_{k}} \circ T^{n_{k}}\right)^{q}\left(\widetilde{z}_{k}\right)\right\}_{i \geq N}$ belong to $R\left(\left(F \circ T^{q}\right)^{p}\left(\Gamma^{\prime}\right)\right)$. On the other hand, the points $\left(F^{m_{k}} \circ T^{n_{k}}\right)^{q}\left(\widetilde{z}_{k}\right)$ belong to $\widetilde{K}_{q} \subset L\left(\left(F \circ T^{q}\right)^{p}\left(\Gamma^{\prime}\right)\right)$, which is a contradiction.

It remains to study case (3) and try to find a contradiction. 
Again, the arguments in the proof of Lemma 10 permit us to construct a line $\Gamma^{\prime}$ of $\mathbb{R}^{2}$ satisfying

- $\Gamma^{\prime} \cap T\left(\Gamma^{\prime}\right)=\emptyset$;

- $T^{-q}\left(\Gamma^{\prime}\right)=F\left(\Gamma^{\prime}\right)$.

Endow $\Gamma^{\prime}$ with the orientation such that $\Gamma^{\prime}<T\left(\Gamma^{\prime}\right)$. As $\Gamma^{\prime}$ is a Brouwer line of $T$, we can find an arc $\Delta_{0}$ that joins a point $z \in \Gamma^{\prime}$ to $T(z)$ whose image is between the two lines $\Gamma^{\prime}$ and $T\left(\Gamma^{\prime}\right)$, and does not intersect the lines but at the extremities. We deduce that $\Delta=\bigcup_{k \in \mathbb{Z}} T^{k}\left(\Delta_{0}\right)$ is the preimage of an essential circle $\delta$ of $\mathbb{A}$. We know that $\operatorname{Rot}_{\text {weak }, \delta}(F)=\{\rho\}$ (the intersection property of $f$ guarantees that it is not empty). By (4) of Proposition 14 and the properties of $\Gamma^{\prime}$, the map $F^{\prime \prime}=F \circ T^{q-1}$ satisfies:

- $F^{\prime \prime}\left(\Gamma^{\prime}\right)=T^{-1}\left(\Gamma^{\prime}\right)<\Gamma^{\prime}$

- $\Delta \subset \bigcup_{k \in \mathbb{Z}} T^{k}\left(\mathrm{Cl}\left(R\left(\Gamma^{\prime}\right)\right) \cap L\left(T\left(\Gamma^{\prime}\right)\right)\right)$;

- $\operatorname{Rot}_{\text {weak }, \delta}\left(F^{\prime \prime}\right)=\{\rho+q-1\} \subset[1,2 q-3]$.

By Lemma 15, the first two items imply that $\operatorname{Rot}_{\text {weak }, \delta}\left(F^{\prime \prime}\right) \subset[-\infty, 0]$, which is contrary to the third item. We have completed the proof.

Replacing the notation $\mathrm{Cl}(\operatorname{Rot}(F))$ in the proof of Theorem 1 by the notation $\operatorname{Rot}_{\text {weak }}(F)$, we have the following similar theorem.

Theorem 18 (Generalization of the line translation theorem). Let $f \in \operatorname{Homeo}_{*}^{\wedge}(\mathbb{A})$ and $F$ be a lift of $f$ to $\mathbb{R}^{2}$. Assume that $\operatorname{Rot}_{\text {weak }}(F)$ is contained in a Farey interval $] \frac{p}{q}, \frac{p^{\prime}}{q^{\prime}}[$. Then, there exists an essential line $\gamma$ in $\mathbb{A}$ such that the lines $\gamma, f(\gamma), \cdots, f^{q+q^{\prime}-1}(\gamma)$ are pairwise disjoint. Moreover, the cyclic order of these lines is the same as the cyclic order of the $q+q^{\prime}-1$ first iterates of a vertical line $\{\theta\} \times \mathbb{R}$ under the rigid rotation with angle $\rho$, for any $\rho \in] \frac{p}{q}, \frac{p^{\prime}}{q^{\prime}}[$.

\section{APPENDIX}

Lemma 19. Let $(X, d)$ be a metric space and $f: X \rightarrow X$ be a continuous map. $A$ positively recurrent point of $f$ is also a positively recurrent point of $f^{q}$ for all $q \in \mathbb{N}$.

Proof. If $z \in \operatorname{Rec}^{+}(f)$, let $O_{i}=\left\{z^{\prime} \in X \mid d\left(z, z^{\prime}\right)<\frac{1}{i}\right\}$ for $i \in \mathbb{N} \backslash\{0\}$. We suppose that $f^{n_{k}}(z) \rightarrow z$ when $k \rightarrow+\infty$. Write $n_{k}=l_{k} q+p_{k}$ where $0 \leq p_{k}<q$. If there are infinitely many $k$ such that $p_{k}=0$, we are done. Otherwise, there are infinitely many $k$ such that $p_{k}=p$ where $0<p<q$. We have $f^{l_{k} q+p}(z) \rightarrow$ $z$ when $k \rightarrow+\infty$. We suppose that $f^{l_{k_{1}} q+p}(z) \in O_{m_{1}}$; then there exists $O_{m_{2}}$ such that $f^{l_{k_{1}} q+p}\left(O_{m_{2}}\right) \subset O_{m_{1}}$. Similarly, there exists $l_{k_{2}}$ and $O_{m_{3}}$ such that $f^{l_{k_{2}} q+p}\left(O_{m_{3}}\right) \subset O_{m_{2}}$. By induction, there is a subsequence $\left(l_{k_{j}}\right)_{j \geq 1}$ of $\left(l_{k}\right)_{k \geq 1}$ and a subsequence $\left\{O_{m_{j}}\right\}_{j \geq 1}$ of $\left\{O_{m}\right\}_{m \geq 1}$ such that $f^{l_{k_{j}} q+p}\left(O_{m_{j+1}}\right) \subset O_{m_{j}}$. Considering the subsequence $\left\{f^{q\left(p+\sum_{j=(t-1) q}^{t q-1} l_{k_{j}}\right)}(z)\right\}_{t \geq 1}$, we are done.

Lemma 20. Let $f \in \operatorname{Homeo}_{*}(\mathbb{A})$ and $F$ be a lift of $f$ to $\mathbb{R}^{2}$. Define the homeomorphism $f_{q}$ of the annulus $\mathbb{A}_{q}=\mathbb{R}^{2} / T^{q}$ lifted by $F$ and write $\pi_{q}: \mathbb{R}^{2} \rightarrow \mathbb{A}_{q}$ for the covering map. If $z \in \operatorname{Rec}^{+}(f)$ and $\widetilde{z} \in \pi^{-1}(z)$, then $\pi_{q}(\widetilde{z}) \in \operatorname{Rec}^{+}\left(f_{q}^{q}\right)$.

Proof. To prove the lemma, we must find two sequences $\left\{i_{n}\right\}_{n \geq 1}$ and $\left\{j_{n}\right\}_{n \geq 1}$ such that $j_{n} \rightarrow+\infty$ and

$$
T^{-q i_{n}} \circ F^{q j_{n}}(\widetilde{z}) \rightarrow \widetilde{z} \quad(\text { when } n \rightarrow+\infty) .
$$


Since $z \in \operatorname{Rec}^{+}\left(f^{q}\right)$, there exist two sequences of integers $\left\{i_{n}\right\}_{n \geq 1}$ and $\left\{j_{n}\right\}_{n \geq 1}$ such that $j_{n} \rightarrow+\infty$ and $T^{-i_{n}} \circ F^{q j_{n}}(\widetilde{z}) \rightarrow \widetilde{z}$ when $n$ tends to $+\infty$. Let $\widetilde{O}_{i}=$ $\left\{\widetilde{z}^{\prime} \in \mathbb{R}^{2} \mid d\left(\widetilde{z}, \widetilde{z}^{\prime}\right)<\frac{1}{i}\right\}$ for $i \in \mathbb{N} \backslash\{0\}$, where $d$ is the Euclidean metric of $\mathbb{R}^{2}$. We suppose that $F^{q j_{n}}(\widetilde{z}) \in T^{i_{n}}\left(\widetilde{O}_{n}\right)$ for every $n$ by considering a subsequence if necessary. Write $i_{n}=q s_{n}+t_{n}$, where $0 \leq t_{n}<q$. If there are infinitely many $n$ such that $t_{n}=0$, we are done. Otherwise, there are infinitely many $n$ such that $t_{n}=p$, where $0<p<q$. We can suppose that $F^{q j_{n}}(\widetilde{z}) \in T^{q s_{n}+p}\left(\widetilde{O}_{n}\right)$ for every $n$ by further considering a subsequence if necessary.

We begin with $F^{q j_{n_{1}}}(\widetilde{z}) \in T^{q s_{n_{1}}+p}\left(\widetilde{O}_{n_{1}}\right)$; then there exists $\widetilde{O}_{n_{2}}$ such that

$$
F^{q j_{n_{1}}}\left(\widetilde{O}_{n_{2}}\right) \subset T^{q s_{n_{1}}+p}\left(\widetilde{O}_{n_{1}}\right) .
$$

Similarly, there exists $\widetilde{O}_{n_{3}}$ such that $F^{q j_{n_{2}}}\left(\widetilde{O}_{n_{3}}\right) \subset T^{q s_{n_{2}}+p}\left(\widetilde{O}_{n_{2}}\right)$. By induction, there is a subsequence $\left\{j_{n_{i}}\right\}_{i \geq 1}$ of $\left\{j_{n}\right\}_{n \geq 1}$, a subsequence $\left\{s_{n_{i}}\right\}_{i \geq 1}$ of $\left\{s_{n}\right\}_{n \geq 1}$ and a subsequence $\left\{\widetilde{O}_{n_{i}}\right\}_{i \geq 1}$ of $\left\{\widetilde{O}_{n}\right\}_{n \geq 1}$ such that $F^{q j_{n_{i}}}\left(\widetilde{O}_{n_{i+1}}\right) \subset T^{q s_{n_{i}}+p}\left(\widetilde{O}_{n_{i}}\right)$. Considering the subsequence

$$
\left\{T^{-q\left(p+\sum_{i=(t-1) q}^{t q-1} s_{n_{i}}\right)} \circ F^{q\left(\sum_{i=(t-1) q}^{t q-1} j_{n_{i}}\right)}(\widetilde{z})\right\}_{t \geq 1},
$$

we are done.

\section{ACKNOWLEDGEMENTS}

The author would like to thank Patrice Le Calvez for helpful conversations and comments. The author also thanks the referees for their careful reading of the text and for their many helpful remarks.

\section{REFERENCES}

[BCL] F. Béguin, S. Crovisier, and F. Le Roux, Pseudo-rotations of the open annulus, Bull. Braz. Math. Soc. (N.S.) 37 (2006), no. 2, 275-306, DOI 10.1007/s00574-006-0013-2. MR.2266384 (2008b:37074)

[BCLP] F. Béguin, S. Crovisier, F. Le Roux, and A. Patou, Pseudo-rotations of the closed annulus: variation on a theorem of J. Kwapisz, Nonlinearity 17 (2004), no. 4, 1427-1453, DOI 10.1088/0951-7715/17/4/016. MR2069713 (2005d:37084)

[C] C. Carathéodory, Über die Begrenzung einfach zusammenhängender Gebiete (German), Math. Ann. 73 (1913), no. 3, 323-370, DOI 10.1007/BF01456699. MR.1511737

[F1] John Franks, Generalizations of the Poincaré-Birkhoff theorem, Ann. of Math. (2) 128 (1988), no. 1, 139-151, DOI 10.2307/1971464. MR951509 (89m:54052)

[F2] John Franks, Area preserving homeomorphisms of open surfaces of genus zero, New York J. Math. 2 (1996), 1-19, electronic. MR1371312 (97c:58123)

[G1] Lucien Guillou, Free lines for homeomorphisms of the open annulus, Trans. Amer. Math. Soc. 360 (2008), no. 4, 2191-2204, DOI 10.1090/S0002-9947-07-04374-7. MR2366979 (2009f:37046)

[G2] Lucien Guillou, Théorème de translation plane de Brouwer et généralisations du théorème de Poincaré-Birkhoff (French), Topology 33 (1994), no. 2, 331-351, DOI 10.1016/0040-9383(94)90016-7. MR1273787(95h:55003)

[Ke] B. Kerékjártó, Vorlesungen über Topologie (I): Flächentopologie, Springer, Berlin, 1923.

[Kw] Jaroslaw Kwapisz, A priori degeneracy of one-dimensional rotation sets for periodic point free torus maps, Trans. Amer. Math. Soc. 354 (2002), no. 7, 2865-2895, DOI 10.1090/S0002-9947-02-02952-5. MR1895207 (2003d:37058)

[Lec1] Patrice Le Calvez, Rotation numbers in the infinite annulus, Proc. Amer. Math. Soc. 129 (2001), no. 11, 3221-3230 (electronic), DOI 10.1090/S0002-9939-01-06165-2. MR1844997 (2002h:37069) 
[Lec2] Patrice Le Calvez, Une version feuilletée équivariante du théorème de translation de Brouwer (French, with English summary), Publ. Math. Inst. Hautes Études Sci. 102 (2005), 1-98, DOI 10.1007/s10240-005-0034-1. MR2217051 (2007m:37100)

[LecY] P. Le Calvez and J.-C. Yoccoz, Suite des indices de Lefschetz des itérés pour un domaine de Jordan qui est un bloc isolant, preprint, 1997.

[Ler1] F. Le Roux, Homéomorphismes de surfaces: théorèmes de la fleur de Leau-Fatou et de la variété stable, Astérisque 292 (2004). MR2068866

[Ler2] F. Le Roux, L'ensemble de rotation autour d'un point fixe d'homéomorphisme de surface, HDR thesis, Université Paris-Sud, (2008).

[M] John N. Mather, Topological proofs of some purely topological consequences of Carathéodory's theory of prime ends, Selected studies: physics-astrophysics, mathematics, history of science, North-Holland, Amsterdam, 1982, pp. 225-255. MR662863 (84k:57004)

[P] A. Patou, Arcs libres d'homéomorphismes en dimension 2, Ph.D. thesis, Université Grenoble 1, (2006).

[S] A. Sauzet, Application des décompositions libres à l'étude des homéomorphismes de surfaces, Ph.D. thesis, Université Paris-Nord (2001).

Chern Institute of Mathematics and LPMC, Nankai University, Tianjin 300071, PeoPLE'S REPUBlic OF CHINA

E-mail address: wangjian@nankai.edu.cn

Current address: Max Planck Institute for Mathematics in the Sciences, Inselstrasse 22, D04103 Leipzig, Germany

E-mail address: jianwang@mis.mpg.de 